Themenheft Nr. 40: CoViD-19 und die digitale Hochschulbildung. Irritationen, Einsichten und Programmatiken

Herausgegeben von Markus Deimann, Marios Karapanos und Klaus Rummler

\title{
CoViD-19 als Katalysator für die digitale Professionalisierung angehender Lehrpersonen?
}

\author{
Analysen am Beispiel des Praxissemesters \\ Franziska Schwabl ${ }^{1}$ iD und Christoph Vogelsang ${ }^{1}$ (D) \\ ${ }^{1}$ Universität Paderborn
}

\section{Zusammenfassung}

Die CoViD-19-Pandemie ab Frühjahr 2020 und die pandemiebedingten Kontaktbeschränkungen hatten erhebliche Auswirkungen auf die studienbegleitenden Praxisphasen im Lehramtsstudium. Studierende, die ihr Praxissemester im Sommersemester 2020 absolvierten, waren in der Folge erheblich von Einschränkungen sowohl während der universitären Begleitveranstaltungen als auch in den Praxisschulen betroffen. Durch die überwiegende Umstellung von Präsenzformaten zu digitalem Lehren und Lernen auf Distanz ist die Annahme naheliegend, dass Studierende vermehrt die Möglichkeit hatten, Erfahrungen mit digitalen Lernformaten zu machen. Anhand einer Sekundäranalyse der Befragung einer Kohorte von Praxissemesterstudierenden der Universität Paderborn ( $N=362)$ kann allerdings gezeigt werden, dass der erwartete Möglichkeitsraum für eine digitale Professionalisierung keineswegs ausgeschöpft bzw. überhaupt genutzt wurde. Vielmehr zeigte sich, dass angehende Lehrpersonen digitale Medien vor allem dann in den eigenen Unterricht einbetten, wenn diese in der mentoriellen Begleitung thematisiert wurde oder während Hospitationen beobachtet werden konnte. Zudem deuten die Analysen an, dass ungünstige Professionalisierungsbedingungen an den Praxisschulen nur bedingt durch die universitären Anteile der Praxisphasen aufgefangen werden konnten. Für die Gestaltung der universitären Lehrpersonenausbildung ergibt sich daher u. a. die Notwendigkeit einer intensiveren Kooperation zwischen den verschiedenen Akteuren der drei Phasen der Lehrpersonenbildung.

CoViD-19 as a Catalyst for the Digital Professionalisation of Prospective Teachers? Analyses on a One-Semester School Internship

\footnotetext{
Abstract

The CoViD-19 pandemic since spring 2020 and the pandemic-related contact restrictions had a considerable impact on the study-accompanying internships in teacher training programmes. Students who completed their internship in the summer semester of 2020
} 
were subsequently significantly affected by the restrictions both during the accompanying seminars at university and during their internships at school. Due to the predominant switch from face-to-face formats to digital teaching and learning, it is reasonable to assume that students had increased opportunities to gain experience with digital learning formats. However, based on the secondary analysis of the survey of a cohort at the University of Paderborn ( $N=362)$, it can be shown that the expected opportunities for digital professionalisation were only limited. Rather, it was shown that (prospective) teachers embedded digital media in their own teaching primarily when this previously was discussed with mentors or could be observed during the observation sessions. In addition, the analyses indicate that unfavourable professionalisation conditions at the practical schools could only be compensated to a limited extent by the university components. For the design of teacher training programmes, therefore there is a need for more intensive cooperation between the actors of teacher training programmes.

\section{Ausgangssituation}

Die CoViD-19-Pandemie im Frühjahr 2020 hatte einen grossen Einfluss auf Bildungsinstitutionen weltweit. Nicht nur in Deutschland wurden zeitweise die Schulen vollständig geschlossen. Auch wenn nach etwa fünf Wochen schrittweise Wiedereröffnungen erfolgten, bestanden viele Restriktionen fort. Lehrpersonen standen vor der Herausforderung, in kurzer Zeit Präsenzunterricht in Formate zum Distanzlehren umzustellen (Fickermann und Edelstein 2020, 10-13). Mittelbar hatten diese Entwicklungen auch einen Einfluss auf die Lehramtsausbildung an den Hochschulen. Zwar hat an den deutschen Hochschulen die Umstellung von der Präsenzlehre auf Distanzformate im Sommersemester 2020 nach ersten Berichten gut funktioniert (Sommer 2020), dennoch wirkten sich die pandemiebedingten Änderungen im Schulbereich auf schulpraktische Studienelemente im Lehramtsstudium aus. Insbesondere begleitete Langzeitpraktika wie das in vielen Bundesländern implementierte Praxissemester waren stark betroffen. Lehramtsstudierende konnten bspw. während der Schulschliessungen nicht oder nur unter Einschränkungen an ihre Praxisschulen zurückkehren und auch universitäre Begleitveranstaltungen fanden überwiegend digital und auf Distanz statt. Solche Praxisphasen haben eine hohe Bedeutung für die Entwicklung der Lehrprofessionalität. Die Diskussionen um die im Zuge der Digitalisierung an (zukünftige) Lehrpersonen gerichtete Erwartungshaltungen bzgl. des Erwerbs digitaler Kompetenzen (KMK 2019) haben nicht zuletzt in der Corona-Pandemie neuen Aufschwung erhalten. Allerdings sind die Ziele der Kultusministerkonferenz zum Umgang mit digitalen Medien bisher kaum verbindlich in der universitären Lehramtsausbildung verankert (Bertelsmann Stiftung et al. 2018). In der Folge haben angehende Lehrpersonen bisher kaum Möglichkeiten, digitale Kompetenzen zu erwerben (Lorenz und Endberg 2019). 
Vor dem Hintergrund der pandemiebedingten Verlagerung der schulischen sowie universitären Lehre ins Digitale ist daher anzunehmen, dass im Frühjahr 2020 in den Praxisphasen des Lehramtsstudiums ein Möglichkeitsraum entstand, der es Studierenden in besonderer Weise ermöglichte, Erfahrungen mit digitalen Lehrformaten zu machen - sowohl als Lehrende (in der Schule), als auch als Lernende (in der Hochschule). Es kann daher vermutet werden, dass die Pandemie gewissermassen als Katalysator wirkte und (vermeintlich) lange ausstehende Digitalisierungsprozesse an Schulen und Universitäten angestossen hat. Die CoViD-19-Pandemie kann in diesem Sinne als Katalysator für die digitale Professionalisierung verstanden werden. Mit anderen Worten, aufgrund der pandemiebedingten Massnahmen wurden an Schulen und Universitäten verstärkt digitale Medien in Lehr-/Lernsettings eingebunden. Daher nehmen wir an, dass die CoViD-19-Pandemie die Ausbildung digitalisierungsbezogener Kompetenzen auch bei angehenden Lehrpersonen vorangetrieben hat. Deren Förderung wird bereits seit den 1980er-Jahren in ordnungspolitischen Dokumenten gefordert, erhält allerdings erst durch die 2016 verabschiedete KMK-Strategie zur «Bildung in der digitalen Welt» Verpflichtungscharakter (Herzig 2021, 4-5).

Der Beitrag widmet sich diesen Annahmen und untersucht, welche Erfahrungen Lehramtsstudierende während der studienbegleitenden Praxisphasen im Frühjahr 2020 tatsächlich machen konnten. Ziel ist es, fundierte Hinweise für Anpassungen dieses praktischen Studienelements zu gewinnen, das sich durch eine komplexe Gemengelage zwischen hochschulischem Lernen, Fragen der technischen Ausstattung an den Schulen und den digitalen Vorerfahrungen der Studierenden auszeichnet. Als empirische Basis werden schriftliche Befragungsdaten von N=362 Lehramtsstudierenden der Universität Paderborn herangezogen, die im Frühjahr 2020 das studienbegleitende Praxissemester absolviert haben.

\section{Auswirkungen der pandemiebedingten Massnahmen auf Praxisphasen im Lehramt}

Die zentralen Lernorte der Praxisphasen - Schulen, Universitäten und Zentren für schulpraktische Lehrpersonenbildung (ZfsL) - waren in unterschiedlichem Ausmass von der Umstellung der Präsenz- auf Distanzformate betroffen. Studierende haben folglich die pandemiebedingten Einschränkungen sowohl als angehende Lehrpersonen, als auch als Lernende und als Forschende erfahren, wobei die verschiedenen Rollen nicht trennscharf sind (Herzig und Wiethoff 2019, 8).

Aufgrund der Aktualität der Ereignisse sind derzeit Studien, die explizit das Erfahren der Praxisphasen während der pandemiebedingten Massnahmen thematisieren, noch rar. Die vorhandenen Berichte beziehen sich entweder auf die Auswirkungen der Einschränkungen in den Schulen (z. B. indem die Erfahrungen der Lehrpersonen, der Schülerinnen und Schüler oder ihrer Eltern thematisiert werden) oder lenken 
ihre Perspektive auf den universitären Kontext, indem sie über die Erfahrungen der Lehrenden und Studierenden berichten. Lernortübergreifende Betrachtungen der Auswirkungen der Pandemie auf studienbegleitende Praxisphasen stehen bisher noch weitestgehend aus. Um den potenziellen Einfluss der Pandemiemassnahmen auf die Praxisphasen abschätzen zu können, betrachten wir daher im Folgenden zunächst die drei Lernorte getrennt. Auf diesen Darstellungen gründen schliesslich die in Unterkapitel 2.4 getroffenen Annahmen zu den Auswirkungen der Pandemie auf das Praxissemester, aus denen schliesslich die Forschungsfragen des Beitrags abgeleitet werden (vgl. Kap. 3).

\subsection{Lernort Schule: Unzureichende technische Infrastruktur trifft auf geringen Einsatz digitaler Medien}

Im schulpraktischen Anteil des Praxissemesters sollen die Studierenden die Möglichkeit haben, die Komplexität des Schulalltags zu erfahren. Lehramtsstudierende der Universität Paderborn verbringen während der rund viermonatigen Praxisphase vier Tage pro Woche in ihren Praxisschulen. Dort haben sie bspw. die Gelegenheit zur Unterrichtshospitation sowie zur Vorbereitung und Durchführung eigener Unterrichtsstunden. Das Praxissemester beginnt an der Universität Paderborn in der Regel Mitte Februar bzw. Mitte September eines Jahres (Herzig und Wiethoff 2019, 12). Aufgrund der pandemiebedingten Schulschliessungen ab Mitte März nahm das Praxissemester im Frühjahr 2020 lediglich etwa vier Wochen seinen gewohnten Gang. Danach waren die Schulen für rund fünf Wochen komplett geschlossen. Anschliessend folgten schrittweise Schulöffnungen, allerdings mit Einschränkungen des Schulbetriebs bis zu den Sommerferien. In dieser Zeit fand Unterricht in unterschiedlicher Form statt häufig wurde Lernen auf Distanz praktiziert (Fickermann und Edelstein 2020, 10-13).

Die Schulschliessungen und die Phasen des Distanzlernens stellten das professionelle Personal an den Schulen vor enorme Herausforderungen. Für Lehramtsstudierende im Praxissemester bedeuteten sie, dass sie nur bedingt die Möglichkeit hatten, den Schulalltag in seiner Komplexität zu erfahren. Die Schulschliessungen wurden als ein Stresstest interpretiert, der die Vernachlässigung der Digitalisierung in den Schulen mehr oder weniger schonungslos aufzeigte (Hoffmann 2020, 95). Kaum eine Schule war auf den abrupten Wechsel von Präsenzunterricht zu Distanzlernen vorbereitet. Daher griffen Schulen überwiegend auf Bewährtes zurück, um das Lernangebot aufrecht zu erhalten: Das am häufigsten für die Kommunikation mit Schülerinnen und Schülern verwendete Medium waren E-Mails, um Aufgaben und Materialien an die Lernenden zu senden. Vergleichsweise selten hingegen wurden Lernplattformen, Clouds, Messengerdienste oder analoge Kommunikationsmedien genutzt (Eickelmann und Drossel 2020, 2-4; Forsa 2020, 26; Huber et al. 2020, 25). Online-Unterricht oder individuelle Gespräche mit den Schülerinnen und Schülern 
fanden ebenfalls nur selten statt (Wößmann et al. 2020, 33). Allerdings zeigen sich Unterschiede zwischen den Schulformen. Während vor allem Grundschulen noch vor Eintritt der pandemiebedingten Schulschliessungen Lernmaterialien in Papierform an die Schülerinnen und Schüler verteilten, stellten Gymnasien und weiterführende Schulen Lernmaterialien eher digital bereit, z. B. per E-Mail oder Clouds. Auch Unterricht in Form von Videokonferenzen fand am häufigsten im gymnasialen Umfeld statt (Huebener, Spieß, und Zinn 2020, 871-72). Die schulspezifischen Unterschiede könnten auf das Alter und die Lernvoraussetzungen der Schülerinnen und Schüler zurückzuführen sein (Huber et al. 2020, 29). Aber auch eine mangelhafte technische Infrastruktur an den Schulen sowie die schlechte technische Ausstattung der Lehrpersonen und Schülerinnen und Schüler bieten Erklärungsansätze (Eickelmann und Drossel 2020, 3; Forsa 2020, 21; Huebener, Spieß, und Zinn 2020, 872).

Hinsichtlich der technischen Ausstattung war Deutschland im europäischen Vergleich bereits vor der Corona-Pandemie abgeschlagen (Eickelmann, Bos, und Labusch 2019, 14-15). In kaum einem anderen Land wurden digitale Medien seltener im Unterricht eingesetzt als in Deutschland. Dazu kommt, dass die Einstellungen der Lehrpersonen gegenüber dem Einsatz von digitalen Medien im Unterricht ambivalent sind (Bos et al. 2016, 150). Wenn digitale Medien eingesetzt werden, dann häufig zur Präsentation von Informationen im Frontalunterricht. Vergleichsweise selten werden sie zur individuellen Förderung einzelner Lernender genutzt. Damit wird das Potenzial digitaler Medien in deutschen Schulen kaum ausgeschöpft. Als ein Grund für die Skepsis gegenüber digitalen Medien wird $u$. a. die mangelnde digitale Kompetenz der Lehrpersonen vermutet: Rund drei Viertel der Lehrpersonen gibt an, während der Lehramtsausbildung nicht gelernt zu haben, wie man digitale Medien im Unterricht nutzt (Eickelmann, Bos, und Labusch 2019, 18). Nach etwa einem halben Jahr Erfahrung mit Lernen auf Distanz geben rund zwei Drittel der befragten Lehrpersonen an, während der Distanzphasen erprobte Ansätze und Instrumente auch zukünftig anwenden zu wollen (Forsa 2020, 36). Studierende in ihren Praxisphasen könnten in Unterrichtshospitationen daher zukünftig vermehrt auch auf Lehrpersonen treffen, die Lerngelegenheiten zum Einsatz digitaler Medien ermöglichen.

\subsection{Lernort Universität: Grosse mediendidaktische Bandbreite und Flexibilität treffen auf ein unzureichendes Angebot zum Austausch}

Neben der Schule ist die Universität ein zentraler Lernort in der Praxisphase. Die Vorbereitungen beginnen dort bereits im Semester vor der eigentlichen Praxisphase, z. B. in Form von bildungswissenschaftlichen und fachspezifischen Vorbereitungsseminaren. Parallel zum an den Schulen absolvierten Praxisteil belegen die Studierenden an der Universität Paderborn einen Tag pro Woche fach- und bildungswissenschaftliche Begleitseminare sowie ein ihr Studienprojekt begleitendes Forschungsseminar (Herzig und Wiethoff 2019, 14). 
Im Frühjahr 2020 fanden universitäre Veranstaltungen überwiegend als Distanzformate statt. Die Bandbreite der praktizierten Möglichkeiten war an den deutschen Hochschulen durchaus heterogen, z. B. asynchrone und synchrone Videokonferenzen, Erklärvideos, Webinare, virtuelle Lehr-/Lernumgebungen. Ersten Berichten zufolge hat die Umstellung von Präsenzlehre auf Distanzformate im Sommersemester 2020 an deutschen Hochschulen gut funktioniert (Sommer 2020). Während noch im Wintersemester 2019/20 lediglich 12 Prozent des Lehrangebots in digitaler Form umgesetzt wurde, fanden im Sommersemester nahezu alle Veranstaltungen digital und auf Distanz statt (Wilde 2020, 3).

Allerdings war das digitale Semester nicht nur für die Lehrenden, die ihre Veranstaltungen innerhalb von wenigen Tagen in digitale Formate bringen mussten, sondern auch für die Studierenden mit Herausforderungen verbunden. Zwar gaben die meisten Studierenden an (78 Prozent), gut mit (mobilen) Rechnern oder anderen Devices ausgestattet zu sein, bei rund einem Viertel allerdings war keine stabile Internetverbindung vorhanden (Lörz et al. 2020). Diese wirkte sich mitunter negativ auf eine reibungslose Teilnahme an Online-Veranstaltungen aus (Traus et al. 2020, 9). Des Weiteren wurde über fehlende und zu wenige Möglichkeiten zum Austausch sowohl mit Kommilitoninnen und Kommilitonen als auch mit den Lehrenden, über Motivations- und Konzentrationsschwierigkeiten beim Lernen zu Hause sowie über eine höher empfundene Arbeitsbelastung berichtet. Zudem erwies es sich - wie bereits in den Schulen - als eine zentrale Herausforderung, dass digitale Lernformate die Fähigkeit zum eigenständigen Lernen voraussetzten, die auch bei Studierenden nicht immer hoch ausgeprägt ist (Wilde 2020, 4-5). Die vorhandenen Selbsteinschätzungen der Studierenden zeichnen aber ein ambivalentes Bild, denn gleichzeitig bewerten Studierende u. a. die während der Pandemiemassnahmen erhöhte Flexibilität in der Arbeitsgestaltung, ausbleibende Anreisewege und das Kennenlernen neuer digitaler Methoden als positiv (Traus et al. 2020, 22). Insgesamt war die Mehrheit der Studierenden mit der pandemiebedingten Umstellung der universitären Lehre zufrieden und honorierte die Reaktionsschnelligkeit ihrer Hochschule (Wilde 2020, 2). Dennoch bewerteten sie mehrheitlich (60 Prozent) die Aussicht auf ein weiteres Online-Semester als unbefriedigend (Traus et al. 2020, 19).

Auch Lehrende bewerteten die Umstellung universitärer Veranstaltungen überwiegend positiv: Langfristig gesehen stehen rund drei Viertel der Hochschullehrenden einer Digitalisierung der Lehre aufgeschlossen gegenüber. Rund die Hälfte der Befragten möchte auch in Zukunft teilweise an digitalen Veranstaltungsformaten festhalten. Zudem geben sie an, dass die Vermittlung digitaler Kompetenzen durch die Corona-Pandemie stärker in den Fokus gerückt ist. Allerdings ist mit Blick auf die universitäre Hochschullehre auch zu konstatieren, dass nicht alle Lehrenden über ausreichend digitale Kompetenzen verfügen. Wenig hilfreich ist dabei, dass fast zwei Drittel Fort- und Weiterbildungsmassnahmen nicht in Anspruch nehmen 
- überwiegend aus Zeitgründen, teils wegen ihrer Einschätzung nach inadäquater Fortbildungsinhalte (Wilde et al. 2020, 2-11). Für die Begleitveranstaltungen im Praxissemester ist die Thematisierung von digitalen Medien (im Unterricht) nicht unerheblich, da anzunehmen ist, dass der Grad, zu dem digitale Medien thematisiert und durch die Studierenden erprobt werden können, einen Einfluss auf die zukünftige Gestaltung ihres eigenen Unterrichts hat.

\subsection{Lernort Zentrum für schulpraktische Lehrpersonenausbildung (ZfsL): Kaum pandemiebedingte Auswirkungen auf die Praxisphasen}

Der dritte Lernort für Studierende im Praxissemester stellt das ZfsL dar. Die Veranstaltungen beginnen in der Ausbildungsregion Paderborn bereits während der vorlesungsfreien Zeit und sind damit den universitären Begleitveranstaltungen vorgelagert. Die Studierenden besuchen am ZfsL allgemein- und fachdidaktische Einführungsveranstaltungen sowie Blockseminare zu verschiedenen Themenbereichen, z. B. Unterrichtsanalyse, kollegiale Fallberatung, Umgang mit Erziehungsproblemen. Mit Ende des schulpraktischen Teils führen Ausbilderinnen und Ausbilder am Lernort Schule Bilanz- und Perspektivengespräche durch, an denen neben den angehenden Lehrpersonen, ihren schulischen Mentorinnen und Mentoren auch Vertreterinnen und Vertreter der universitären Begleitveranstaltungen teilnehmen (können) (Herzig und Wiethoff 2019, 13).

Die Einschränkungen im Sommersemester 2020 betrafen auch die ZfsL. Zwar gibt es z. B. vom Ministerium für Schule und Bildung des Landes Nordrhein-Westfalen veranlasste Handlungsempfehlungen und Impulspapiere, die an Lehrpersonen sowie Seminarausbilderinnen und Seminarausbilder an den ZfsL adressiert sind (Krommer, Wampfler, und Klee 2020), allerdings fehlen derzeit noch Studien, die das Ausmass pandemiebedingter Einschränkungen untersuchen. Für die Ausbildungsregion Paderborn kann festgehalten werden, dass die CoViD-19-Pandemie kaum Auswirkungen auf die ZfsL-Veranstaltungen hatte. Da die erwähnten Blockveranstaltungen noch vor den Kontaktbeschränkungen in Präsenz stattfanden, waren einzig die Bilanz- und Perspektivgespräche am Ende der Praxisphase betroffen. Diese fanden in der Ausbildungsregion Paderborn nur in Ausnahmefällen in Präsenz statt.

\subsection{Auswirkung der pandemiebedingten Massnahmen auf die Professionalisierung angehender Lehrpersonen}

Während die Kontaktbeschränkungen kaum Folgen für die Veranstaltungen des ZfsL in der Ausbildungsregion Paderborn hatten, waren die Auswirkungen auf den schulpraktischen Teil und auf die universitären Begleitveranstaltungen doch erheblich. Die Umstellung von Präsenzformaten zu digitalem Lernen auf Distanz war für 
Studierende im Praxissemester durchaus eine Herausforderung. Zwar zählen die heutigen Studierenden zu den Digital Natives (Prensky 2001) und ihnen wird aufgrund der im Alltag praktizierten Nutzung von digitalen Werkzeugen ein hohes Mass an digitalen Kompetenzen zugesprochen. Allerdings sind die Medienkompetenzen angehender Lehrpersonen heterogen und können nicht pauschal vorausgesetzt werden (Raabe-Maticevic und Veith 2020, 104). Studierende nutzen zwar häufig digitale Medien, allerdings wenig gezielt zu Lernzwecken (Persike und Friedrich 2016). Meist werden digitale Medien in der Freizeit zu Unterhaltungszwecken oder zu gestalterischen Zwecken eingesetzt. Im Studium hingegen greifen Studierende häufiger auf digitale Standardmedien wie E-Mails, Textverarbeitungsprogramme oder die Lernplattformen der Hochschulen zurück (Zawacki-Richter 2015). Insbesondere die Gruppe der Lehramtsstudierenden scheint nach Selbstauskünften digitale Medien sowohl in Lehrveranstaltungen als auch ausserhalb weniger zu nutzen (Schmidt et al. 2017, 6). Ein erheblicher Anteil der Studierenden eignet sich gestalterische und technische Kompetenzen sogar erst während des Lehramtsstudiums an (Niesyto 2020, 89). Dies geschieht nicht ausschliesslich in universitären Lehrveranstaltungen. Auch die schulischen Praxisphasen sind eine Gelegenheit, den Einsatz digitaler Medien im Unterricht zu erproben. Insgesamt kommt derzeit allerdings der Einsatz digitaler Medien - unabhängig von den Pandemiemassnahmen - sowohl an den deutschen Schulen als auch in der universitären Lehrpersonenausbildung zu kurz (Eickelmann, Bos, und Labusch 2019, 14-18).

Auf Basis eines systematischen Forschungsreviews identifizierten Tondeur et al. (2012) zwölf Schlüsselstrategien, die die Vorbereitung angehender Lehrpersonen für die Integration digitaler Medien in schulischen Handlungsfeldern positiv beeinflussen und die als Heuristik verwendet werden können, um Auswirkungen der CoViD19-Pandemie auf die Entwicklung digitalisierungsbezogener Kompetenzen näher zu betrachten. Diese umfassen auf Ebene einzelner Ausbildungselemente (1) die adäquate Relation von Theorie und Praxis des Medieneinsatzes, (2) die wichtige Rolle von Lehrpersonenausbildenden als Vorbilder für den Medieneinsatz, (3) die Reflexion eigener Ein- und Vorstellungen zur Integration digitaler Medien durch die Studierenden, (4) die Möglichkeit, digitale Medien eigenständig zu erstellen und einzusetzen, (5) kollaborative Zusammenarbeit mit Mitstudierenden bei der Integration digitaler Medien, (6) die Möglichkeit abgestufte, authentische Erfahrungen beim Medieneinsatz zu machen und (7) kontinuierliches Feedback bei der Entwicklung digitalisierungsbezogener Kompetenzen. Auf institutioneller Ebene sind wichtige Strategien (8) eine bewusste Planung und Zielsetzungen innerhalb der Ausbildungsinstitutionen bzgl. des Aufbaus digitaler Kompetenzen, (9) digitalisierungsbezogene Kooperationen zwischen verschiedenen Ausbildungsinstitutionen, (10) die Weiterbildung der Ausbildenden in den Institutionen, (11) die Bereitstellung technischer Ressourcen und (12) systemische Weiterentwicklungen der Institutionen zur Unterstützung eines graduellen Kompetenzaufbaus. 
Da im Frühjahr 2020 sowohl die schulpraktischen Teile als auch die universitären Begleitveranstaltungen in den Praxisphasen in weiten Teilen von Präsenzformaten zu digitalem Lernen auf Distanz umgestellt wurden, ist anzunehmen, dass sich hierdurch für Studierende vermehrt Möglichkeiten boten, digitale Medien sowohl im schulischen als auch universitären Kontext zu thematisieren, zu nutzen und selbstständig zu erproben. Denn obwohl die Umstellung für die meisten Schulen und Universitäten eine Herausforderung darstellte, zeigen erste Berichte, dass digitale Medien in diesem Zeitraum häufiger zum Einsatz kamen als vor der Pandemie (vgl. Unterkap. 2.1 und 2.2). Bezogen auf den Lernort Schule kann vermutet werden, dass sie aufgrund der Anpassungen im Distanzunterricht vermehrt Lehrpersonen als Vorbilder des Medieneinsatzes erleben konnten und auch selbst vermehrt selbst digitale Medien in einem authentischen Setting implementieren konnten bzw. mussten. Idealerweise erhielten sie auch Feedback hierzu durch Lehrpersonen (Schlüsselstrategien 2, 4, 6 und 7). Bezogen auf den Lernort Universität bestand zum einen ebenfalls die Möglichkeit, selbst mit digitalen Medien als Lernende authentische Erfahrungen zu machen. Zum anderen kann aber auch vermutet werden, dass digitale Medien in den Begleitveranstaltungen auch theoretisch-konzeptionell thematisiert wurden und idealerweise auch in Bezug zur schulischen Anwendungspraxis reflektiert wurden (auch im Sinne eines Feedbacks). Angehende Lehrpersonen orientieren sich in der Gestaltung des eigenen Unterrichts mittels digitaler Medien letztlich auch am in universitären Begleitveranstaltungen beobachteten Einsatz digitaler Medien im Sinne eines Vorbilds (Schlüsselstrategien 1, 4 und 7). Für den Lernort $Z f_{s} L$ ist hingegen zu vermuten, dass vor dem Hintergrund der geringen Auswirkungen während der CoViD-19-Pandemie kein zusätzlicher Einfluss auf ihre digitale Professionalisierung ausging.

\section{Fragestellungen}

Dieser Beitrag untersucht, inwiefern das Praxissemester im Frühjahr 2020 als der erwartete Katalysator für eine Professionalisierung im Umgang mit digitalen Medien dienen konnte. Ziel ist es, Hinweise für Anpassungen dieses Studienelements zu gewinnen, das sich durch eine komplexe Gemengelage zwischen hochschulischem Lernen, Fragen der technischen Ausstattung an Schulen und den digitalen Vorerfahrungen der Studierenden auszeichnet. Es ergeben sich folgende Forschungsfragen:

- Über welche Vorerfahrungen mit digitalen Werkzeugen verfügten Lehramtsstudierende zu Beginn des Praxissemesters?

- Welche Erfahrungen konnten Studierende an den Lernorten Schule und Universität machen und inwiefern hatten sie die Möglichkeit, digitale Medien selbst einzusetzen? 
- Welche Zusammenhänge bestehen zwischen Vorerfahrungen mit digitalen Werkzeugen und der Nutzung von Lerngelegenheiten?

- Welchen Einfluss hat die technische Infrastruktur an den Schulen auf die Erfahrungen der Studierenden?

Da das Ziel Aussagen über die Gruppe der Lehramtsstudierenden zumindest an einem Ausbildungsstandort sind, werden primär quantitativ-deskriptive Analysen vorgenommen.

\section{Untersuchungsdesign}

Zur Untersuchung der Forschungsfragen werden Daten herangezogen, die im Rahmen der regelmässigen Evaluation des Praxissemesters an der Universität Paderborn erhoben werden. Hierzu bearbeiten die Studierenden in einem Prä-Post-Design zu Beginn und am Ende des Praxissemesters zwei umfangreiche Online-Fragebögen, in denen ihre Einschätzungen zu vielen Aspekten des Praxissemesters erfragt werden. Für diesen Beitrag werden in einer Sekundäranalyse jeweils spezifische Teile der Fragebögen zu beiden Messzeitpunkten herangezogen. Die eingesetzten Instrumente wurden daher nicht speziell auf diese Untersuchung optimiert bzw. konnten im laufenden Semester nur teilweise angepasst werden. Die Auswertung erfolgt daher eher explorativ.

\subsection{Instrumente}

Zu Beginn des Praxissemesters wurden die Studierenden hinsichtlich ihres Mediennutzungsverhaltens und ihrer lernbezogenen Vorerfahrungen mit digitalen Werkzeugen befragt (Vogelsang et al. 2019). Hierzu wurden sie gebeten, auf vierstufigen Items (1=(fast) nie, 4=sehr oft; nur Endpunkte benannt) anzugeben, wie häufig sie digitale Medien für bestimmte Zwecke nutzen (17 Items). Mit ähnlichen Items wurden die Studierenden zudem befragt, wie oft sie lernbezogene Vorerfahrungen mit digitalen Medien während ihrer Schulzeit und in ihrem bisherigen Studium gemacht haben. Hierzu wurde je Item eine lernbezogene Nutzung digitaler Medien sowohl für die Schulzeit als auch für das Studium beschrieben (je 19 Items). In Abb. 1 sind beispielhaft verwendete Items dargestellt. 
I ch selbst nutze digitale Medien...

$\begin{array}{llll}\text { (fast) nie } \quad \ldots & \ldots & \text { sehr oft }\end{array}$

...um Informationen zu bestimmten Themen zu suchen.

...um (online und/oder offline) spiele $z u$ spielen.

W ährend meiner Schulzeit habe ich...

\begin{tabular}{|c|c|c|c|c|}
\hline & (fast) nie & $\ldots$ & $\ldots$ & sehr oft \\
\hline $\begin{array}{l}\text {..Tabellenkalkulationsprogramme (z.B. } \\
\text { Excel) zur Bearbeitung von Aufgaben } \\
\text { genutzt. }\end{array}$ & $\bigcirc$ & $\bigcirc$ & $\mathrm{O}$ & 0 \\
\hline $\begin{array}{l}\text {...mit Hilfe von digitalen Medien Texte } \\
\text { verfasst. }\end{array}$ & 0 & $\bigcirc$ & & \\
\hline
\end{tabular}

Abb. 1.: Itembeispiele - Mediennutzungsverhalten und lernbezogene Vorerfahrungen.

Zur Einschätzung der digitalen Lerngelegenheiten am Lernort Schule wurde zum zweiten Messzeitpunkt zunächst erfragt, ob die Praxisschule mit bestimmten (digitalen) Medien ausgestattet ist (8 Items). Hierbei wurde zwischen einer festinstallierten Ausstattung und einer mobilen Ausstattung unterschieden. Weiterhin wurden die Studierenden in jedem ihrer Unterrichtsfächer auf einer vierstufigen Skala danach gefragt, in welchem Ausmass sie den Einsatz digitaler Medien im Unterricht beobachten konnten (1=weniger als zweimal im Monat; $2=$ ungefähr einmal in der Woche; 3=mehr als einmal in der Woche; 4=mit wenigen Ausnahmen täglich). Hierbei wurde unterschieden zwischen Unterricht in Präsenz (11 Items) und Unterricht auf Distanz (9 Items). Im selben Format wurde ebenfalls erfragt, in welchem Ausmass die Studierenden diese digitalen Medien im von ihnen selbst gestalteten Unterricht eingesetzt haben. In Abb. 2 sind beispielhaft verwendete Items dargestellt. 


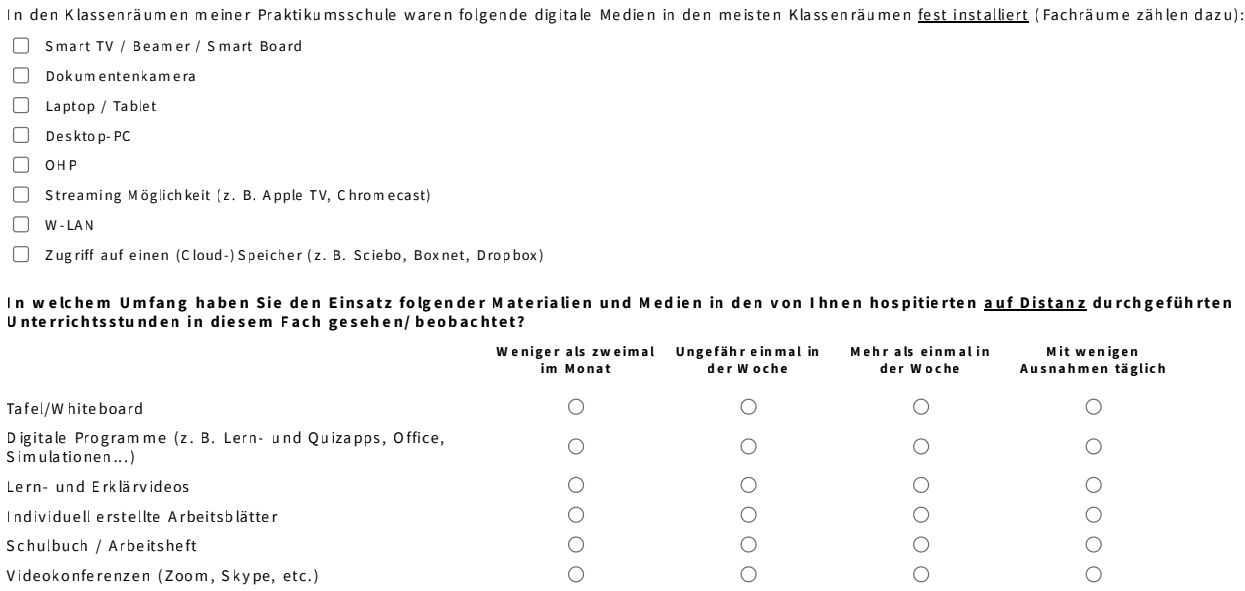

Abb. 2.: Itembeispiele - Ausstattung und Lernerfahrungen mit digitalen Medien am Lernort Schule.

Zur Abschätzung, in welchem Umfang das Lernen mit digitalen Medien Gegenstand der begleitenden universitären Veranstaltungen und der schulischen Begleitung durch Mentorinnen und Mentoren waren, wurden vierstufige Likert-Items herangezogen, mit denen nach dem Praxissemester für jedes Fach erfragt wurde, in welchem Umfang über bestimmte Themen gesprochen wurde (1=sehr wenig; 2=wenig, $3=$ oft $4=$ sehr oft). In Abb. 3 sind beispielhaft verwendete Items dargestellt.

\begin{tabular}{|c|c|c|c|c|}
\hline & sehrwenig & wenig & oft & sehroft \\
\hline Strukturierung von Unterricht & O & O & O & O \\
\hline $\begin{array}{l}\text { Einbettung einer Unterrichtsstunde in } \\
\text { eine Unterrichtsreihe }\end{array}$ & O & O & O & O \\
\hline Fachliche Inhalte & O & O & O & O \\
\hline $\begin{array}{l}\text { Didaktische Reduktion von fachlichen } \\
\text { Inhalten }\end{array}$ & O & O & O & O \\
\hline Aufgabenstellungen und Arbeitsaufträge & 0 & 0 & O & O \\
\hline (Kern-) lehrplan & O & 0 & O & O \\
\hline $\begin{array}{l}\text { Lernvoraussetzungen (z.B. Vorwissen, } \\
\text { allgemeine Leistungsfähigkeit...) }\end{array}$ & $\mathrm{O}$ & $\mathrm{O}$ & O & $\mathrm{O}$ \\
\hline $\begin{array}{l}\text { Umgang mit Heterogenität (z.B. } \\
\text { Differenzierung, Inklusion) }\end{array}$ & O & O & O & O \\
\hline $\begin{array}{l}\text { Aktivierung von Schülerinnen und } \\
\text { Schülern }\end{array}$ & O & O & O & O \\
\hline Motivation & O & O & O & O \\
\hline $\begin{array}{l}\text { Digitale Medien als Hilfsmittel zur } \\
\text { Organisation oder } \\
\text { Unterrichtsvorbereitung }\end{array}$ & O & 0 & 0 & O \\
\hline
\end{tabular}

Abb. 3.: Itembeispiele - Digitale Medien als Thema der mentoriellen und universitären Begleitung. 


\subsection{Auswertung}

Die Antworten der Studierenden wurden zunächst in Zahlenwerte umgewandelt und bereinigt. Dabei wurden Werte aus der Analyse ausgeschlossen, wenn sich bspw. Ankreuzmuster feststellen liessen, ebenso, um Verzerrungen einzuschränken, wenn Personen innerhalb eines Itembereichs aufgrund der Freiwilligkeit der Befragung zu wenig Antworten angegeben haben $(<60 \%)$. In Analysen wurden Werte paarweise eingeschlossen, weshalb die Anzahl der zu Grunde liegenden Studierendenangaben variiert. Dies wird in der Darstellung jeweils ausgewiesen.

Für die Items zur Erfassung der Lerngelegenheiten, die jeweils für jedes von den Studierenden unterrichtete Fach erfragt wurden, wurden anschliessend itemweise Mittelwerte über alle Fächer gebildet, um die Gesamtheit der Erfahrungen abzubilden. Die Auswertung erfolgt anschliessend zunächst deskriptiv für die Gesamtgruppe.

Um in komprimierter Form Zusammenhänge zwischen Vorerfahrungen, Ausstattung und Lerngelegenheiten untersuchen zu können, wurden die einzelnen Items zu Skalen zusammengefasst. Bzgl. des Mediennutzungsverhaltens und der lernbezogenen Vorerfahrungen erfolgte die Skalenbildung in Anlehnung an Vogelsang et al. (2019). Bezogen auf die Erfahrungen an den Praxisschulen sowie in der universitären und mentoriellen Begleitung wurden jeweils alle Items, die eine Erfahrung bezogen auf digitale Medien bezeichnen, zu einer Gesamtskala zusammengefasst. Die sich ergebenen Werte können i. S. einer Intensität von Lernerfahrungen interpretiert werden. Bei der Interpretation muss allerdings berücksichtigt werden, dass es sich um sehr unterschiedliche Skalen handelt. Zur besseren Übersicht erfolgt eine Beschreibung der Reliabilitäten im jeweiligen Abschnitt der Ergebnisdarstellung. Zusammenhänge zwischen den Skalen wurden mittels bivariater Korrelationen analysiert, Unterschiede zwischen Gruppen mit Hilfe von einfaktoriellen ANOVAs.

\subsection{Stichprobe}

Für das Praxissemester im Frühjahr/Sommer 2020 liegen Ergebnisse von N=362 Studierenden vor. Das entspricht 95.6\% der Gesamtkohorte.

\begin{tabular}{|l|c|}
\hline Gender & Anteil (in \%) \\
\hline weiblich & 70.6 \\
\hline männlich & 29.4 \\
\hline divers & --- \\
\hline keine Angabe & 0.3 \\
\hline
\end{tabular}




\begin{tabular}{|l|c|}
\hline LA-Studiengang für... & Anteil (in \%) \\
\hline Grundschule & 20.4 \\
\hline Haupt-, Real-, Sekundar- und Gesamtschule & 22.1 \\
\hline Gymnasien und Gesamtschule & 28.7 \\
\hline sonderpädagogische Förderung & 13.5 \\
\hline Berufskolleg & 15.2 \\
\hline
\end{tabular}

Tab. 1.: Stichprobe.

Die Verteilung der Studierenden hinsichtlich der Studiengänge und des Geschlechts unterscheidet sich nicht signifikant von den Verteilungen vorheriger Jahrgänge. Die Stichprobe kann daher als repräsentativ für das Praxissemester in der Ausbildungsregion Paderborn interpretiert werden. Auf die Frage «Wurden Sie während der Corona-Pandemie von Ihrer/m Mentor/in mit einbezogen?» antworteten 122 Studierende (33.8\% der Stichprobe) mit «Nein«.

\section{Ergebnisse}

\subsection{Nutzungsverhalten und lernbezogene Vorerfahrungen im Umgang mit digitalen Medien}

Nachfolgend sind die Antworten der Studierenden zum Mediennutzungsverhalten (Abb. 4), zu lernbezogenen Vorerfahrungen im Umgang mit digitalen Medien in der Schulzeit (Abb. 5) und während des Studiums (Abb. 6) visualisiert. Angegeben sind itemweise die prozentualen Anteile der Antworten, absteigend sortiert nach Häufigkeit. Zur besseren Übersicht wurden die Nutzungsszenarien verschlagwortet. Die Antworten beziehen sich auf den ersten Messzeitpunkt. 


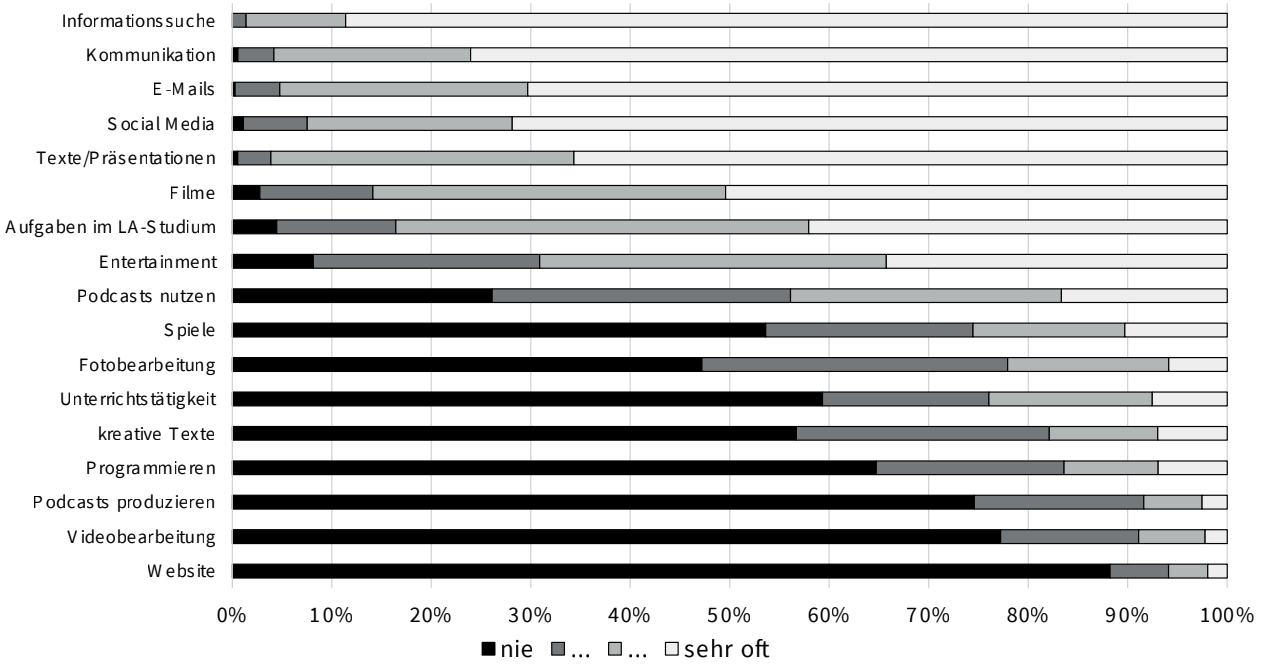

Abb. 4.: Mediennutzungsverhalten ( $\mathrm{N}=361)$.

Bezogen auf die Ergebnisse zum Mediennutzungsverhalten fällt auf, dass insbesondere die Nutzung digitaler Medien zu Kommunikationszwecken (z. B. Social Media) und zu Standardanwendungen des Studiums (z. B. Informationssuche) am häufigsten genannt werden. Eher gestaltende Nutzungsformen (z. B. Erstellung einer eigenen Website) werden nur von wenigen Studierenden angegeben. Zur besseren Übersicht wurden aus den Items die beiden Subskalen unterhaltungsorientiere Mediennutzung (Cronbach's $\alpha=0.64, \mathrm{MW}=3.41, \mathrm{SD}=0.52$ ) und gestaltende Mediennutzung (Cronbach's $\alpha=0.70, M W=1.68, S D=0.44$ ) gebildet (Vogelsang et al. 2019). Hier zeigt sich ein starkes Übergewicht unterhaltungsorientierter Nutzung. Die Skalen weisen allerdings nur eine mittlere interne Konsistenz auf. 


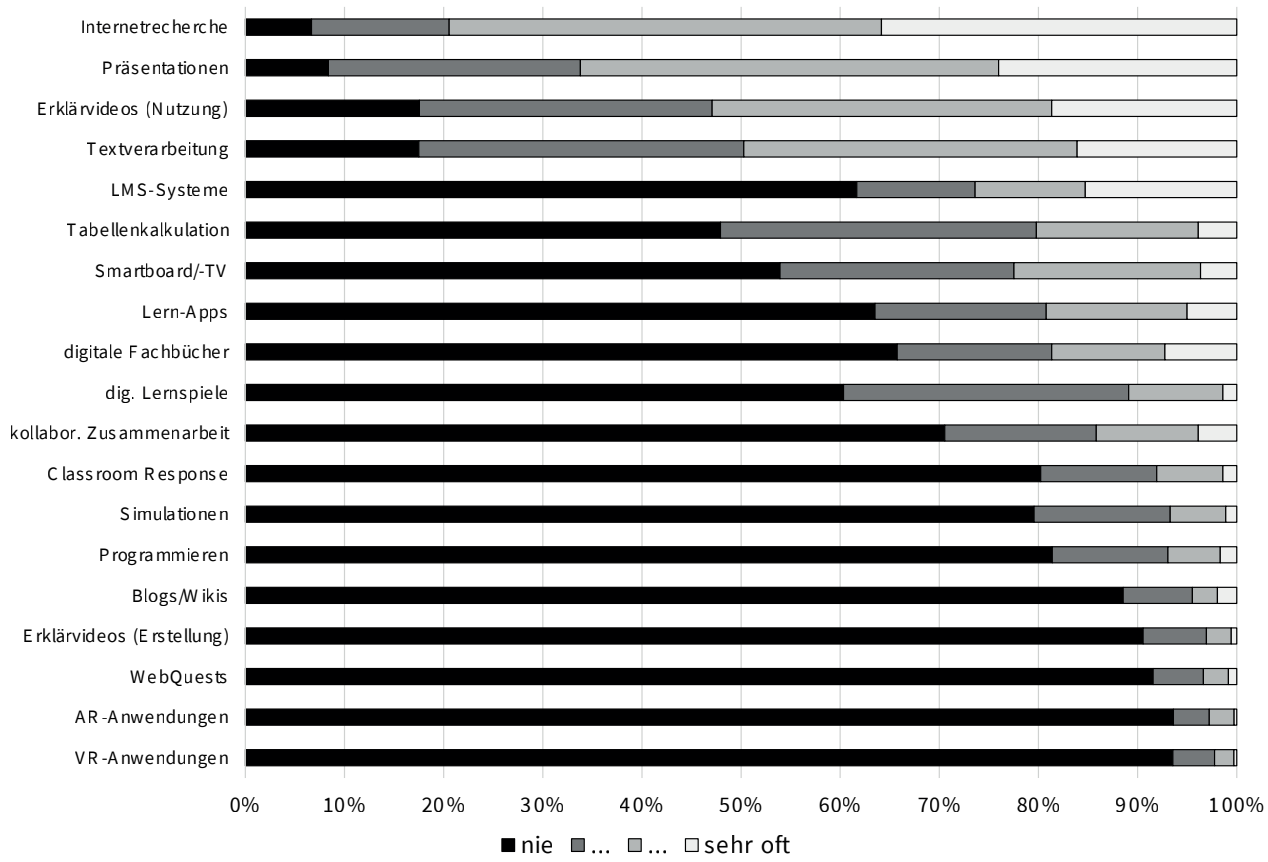

Abb. 5.: Lernbezogene Vorerfahrungen mit digitalen Medien - Schule ( $N=361)$.

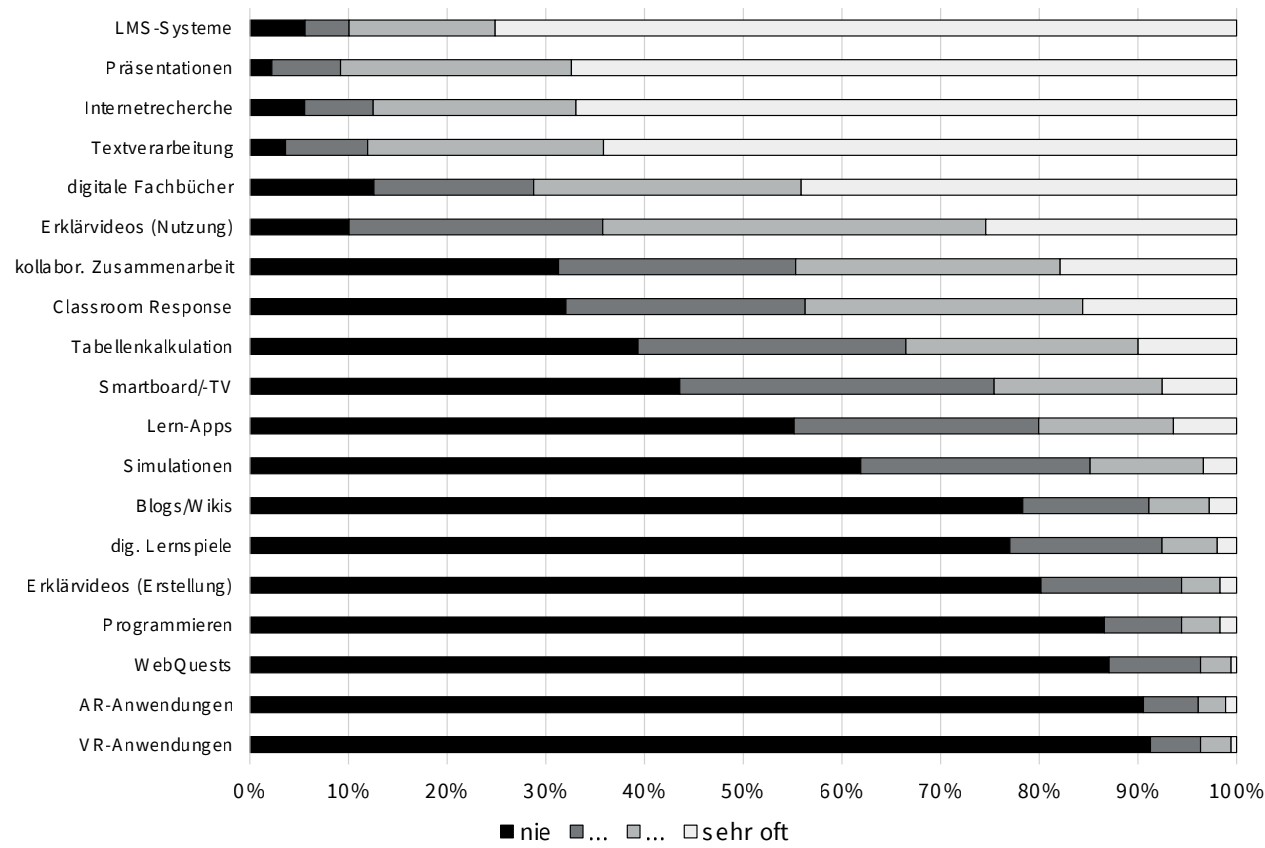

Abb. 6.: Lernbezogene Vorerfahrungen im Umgang mit digitalen Medien - Studium ( $N=361)$. 
Auffallend ist das geringe Ausmass an schulischen Vorerfahrungen mit Ausnahme der Standardanforderungen Textverarbeitung, Präsentationen und Internetrecherche sowie der Nutzung von Erklärvideos. Im Studium bestehen zudem überwiegend Erfahrungen zur Nutzung von Lernmanagementsystemen und digitalen Fachbüchern. Auch hier wurde zur besseren Übersicht jeweils eine Skala der Erfahrungsintensität für jeden Bereich gebildet (Vogelsang et al. 2019), in die einfach alle Items einbezogen wurden. Dabei ergibt sich zwischen den schulbezogenen Vorerfahrungen (Cronbach's $\alpha=0.88, M W=1.68, S D=0.44$ ) und den Vorerfahrungen im Studium (Cronbach's $\alpha=0.82$, $M W=2.13, S D=0.40$ ) durchschnittlich ein Mehr an Erfahrungen im Studium.

\subsection{Lerngelegenheiten am Lernort Schule}

Zur Einschätzung der technischen Voraussetzungen an den Praxisschulen wird in Abb. 7 prozentual dargestellt, über welche Ausstattung die Schulen nach Studierendenaussagen verfügen, unterschieden nach festinstallierter und mobiler Ausstattung (z. B. als Medienwagen).

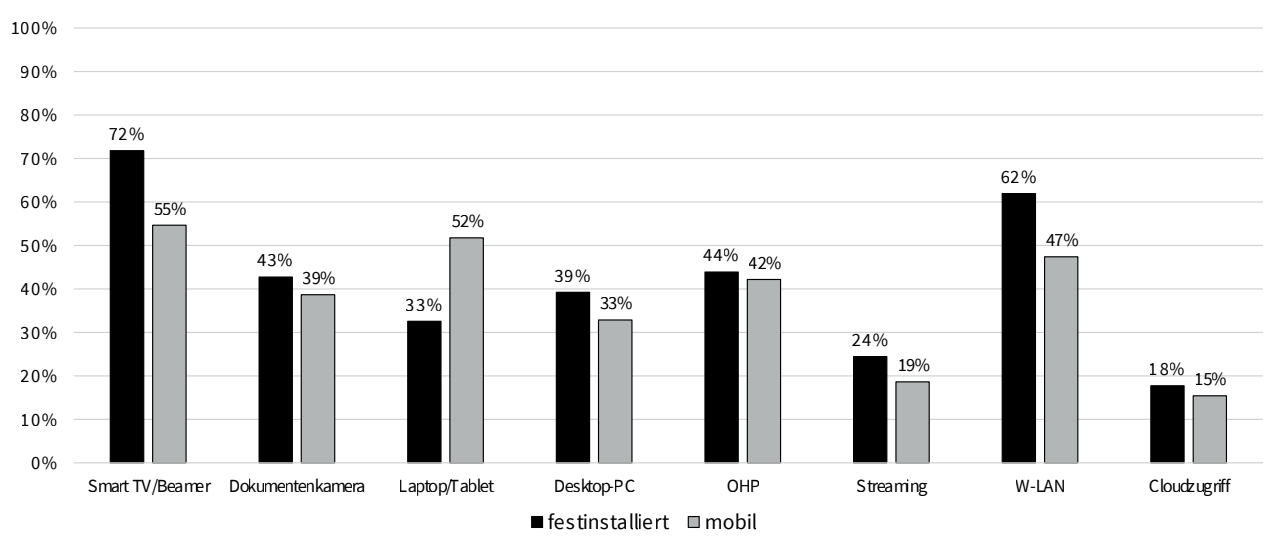

Abb. 7.: Medienausstattung der Praxissemesterschule ( $\mathrm{N}=344)$.

Die häufigsten berichteten Medienarten sind festinstallierte Beamer/SmartTVs bzw. W-LAN-Zugänge, jeweils zu gut einem Drittel. Laptops/Tablets waren in ca. der Hälfte der Schulen mobil verfügbar.

Die Antworten der Studierenden bzgl. der Erfahrungen mit (digitalen) Medien an den Schulen sind in Abb. 8 bezogen auf Hospitationen und in Abb. 9 bezogen auf den Einsatz im eigenen Unterricht itemweise als Boxplots dargestellt. Dabei wird farblich zwischen Erfahrungen im Präsenz- und im Distanzunterricht unterschieden. Manche Medienarten wurden nur für eine Form erfragt, da ihr Einsatz für die jeweils andere als sehr unwahrscheinlich angenommen wurde (z. B. Videokonferenzen im Präsenzunterricht). 


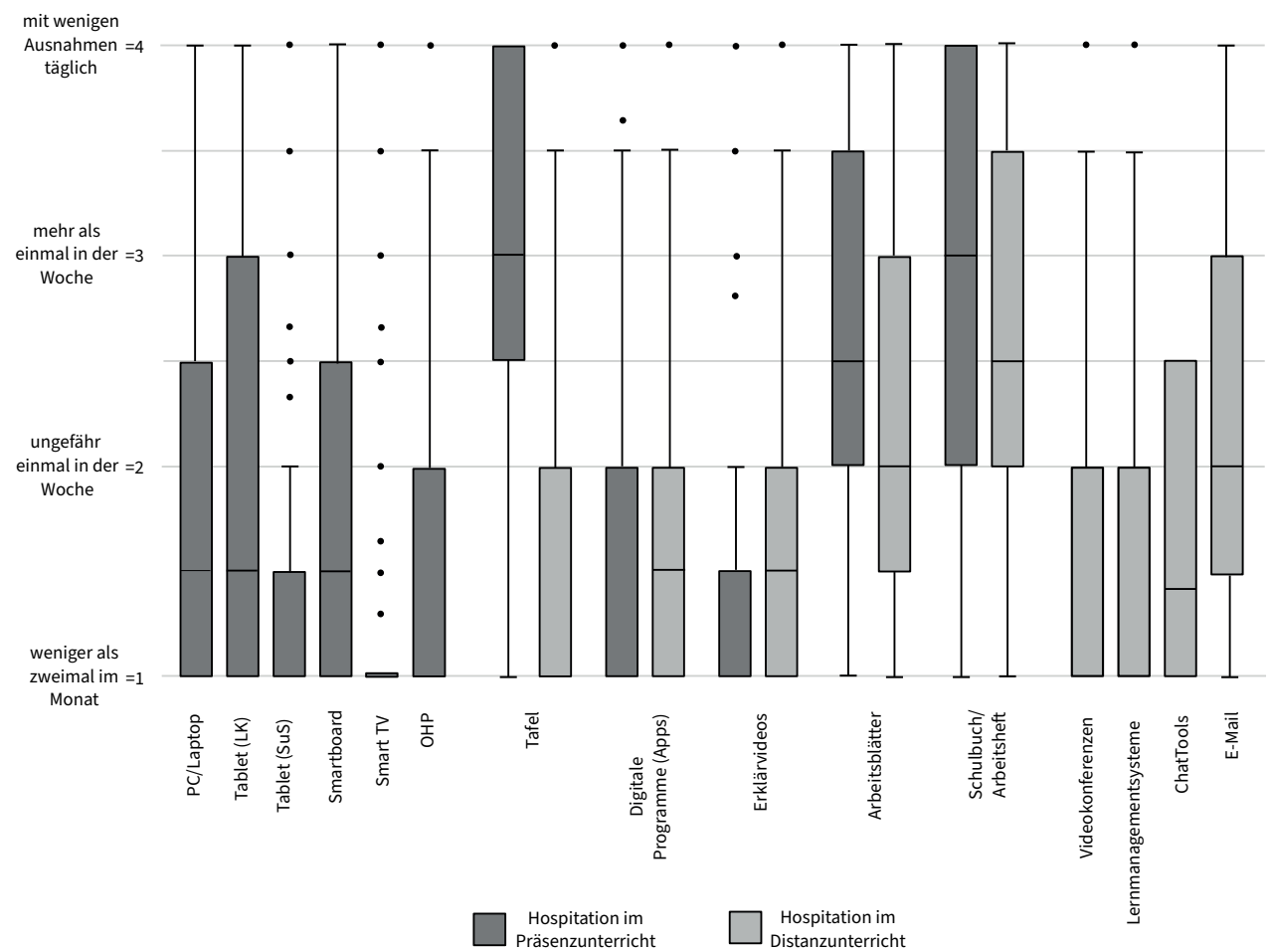

Abb. 8.: Lerngelegenheiten mit Medien - Hospitation im Präsenz- und Distanzunterricht ( $N=265$ 302, je nach Item).

Bei der Hospitation in Präsenz- und Distanzunterricht wurde der Einsatz analoger Medien weitaus häufiger beobachtet, als der Einsatz digitaler Medien. Die durchschnittlich häufigste Hospitation eines digitalen Mediums im Präsenzunterricht betrifft den Tableteinsatz durch die Lehrperson, im Distanzunterricht den Einsatz von E-Mails. Um die Beobachtungsintensität des digitalen Medieneinsatzes in Hospitationen grob zu quantifizieren, wurden zwei Skalen gebildet, die die jeweiligen digitalen Medien umfassen: Hospitation digitalen Medieneinsatzes im Präsenzunterricht (Cronbach's $\alpha=0.72, \mathrm{MW}=1.66, \mathrm{SD}=0.55$ ) und Hospitation digitalen Medieneinsatzes im Distanzunterricht (Cronbach's $\alpha=0.78, \mathrm{MW}=1.75, \mathrm{SD}=0.75$ ). Da beide Skalen unterschiedliche Items enthalten, sind sie nicht direkt vergleichbar. 


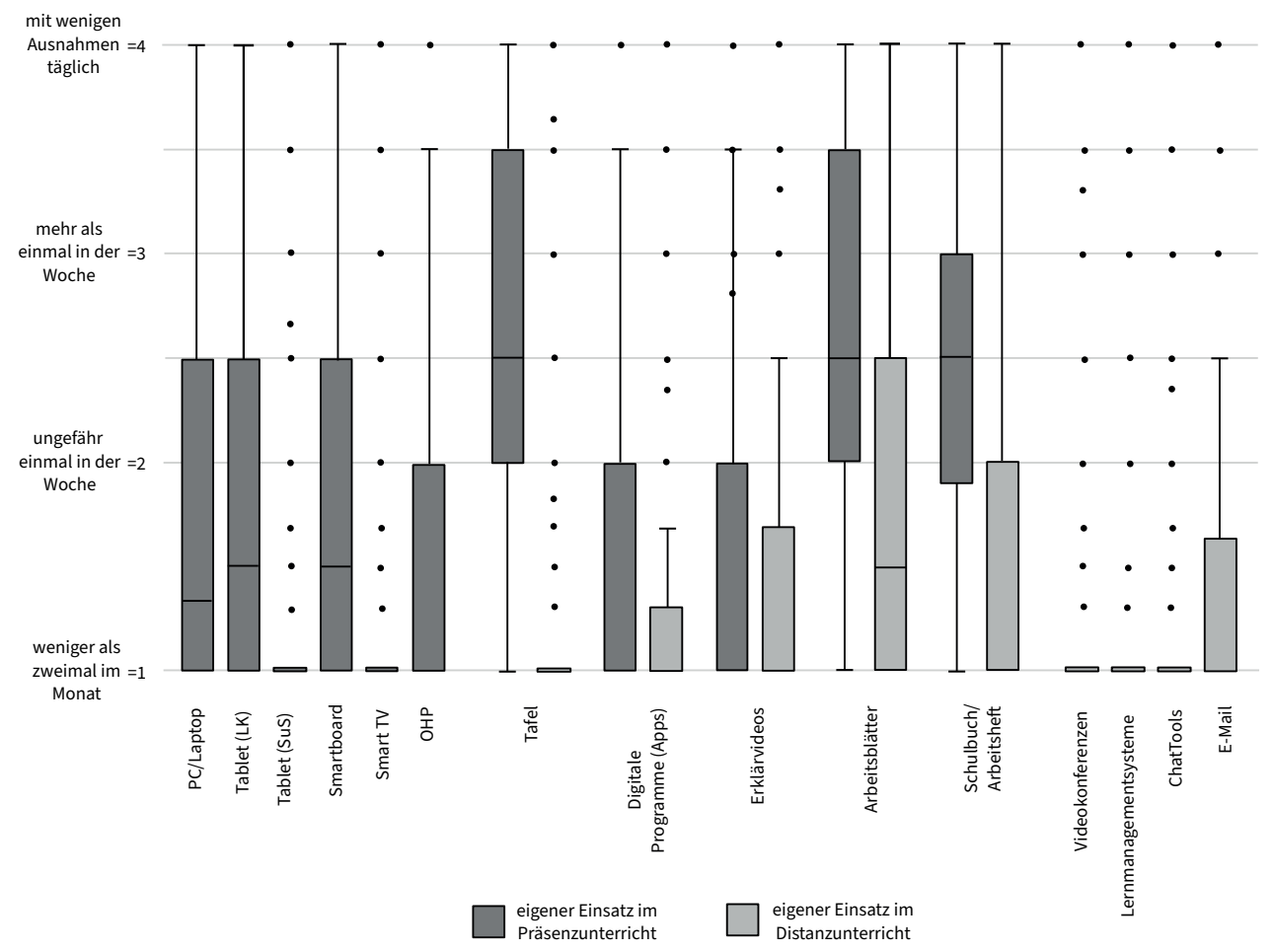

Abb. 9.: Lerngelegenheiten mit Medien - eigener Einsatz im Präsenz- und Distanzunterricht ( $N=267-321$, je nach Item).

Deskriptiv fällt auf, dass auch beim selbst durchgeführten Unterricht der Einsatz analoger Medien weitaus häufiger angegeben wurde. Der durchschnittlich häufigste Einsatz eines digitalen Mediums im Präsenzunterricht - bei insgesamt sehr niedrigem Niveau - ist der Einsatz eines Tablets oder eines Smartboards, im Distanzunterricht der Einsatz von Erklärvideos. Es wurden zur Quantifizierung zwei Skalen analog zu den Hospitationserfahrungen gebildet: Eigener digitaler Medieneinsatz im Präsenzunterricht (Cronbach's $\alpha=0.77, \mathrm{MW}=1.51, \mathrm{SD}=0.53$ ) und Eigener digitaler Medieneinsatz im Distanzunterricht (Cronbach's $\alpha=0.87, \mathrm{MW}=1.33, \mathrm{SD}=0.54$ ).

\subsection{Universitäre Begleitveranstaltungen und mentorielle Begleitung}

Zur Einschätzung in welcher Intensität bestimmte schulbezogene bzw. bildungswissenschaftliche Themenbereiche in der universitären und mentoriellen Begleitung im Praxissemester thematisiert wurden, sind die Antworten der Studierenden für eine Auswahl an Themenbereichen in Abb. 10 als Boxplots dargestellt, farblich unterschieden nach der Thematisierung in universitären Begleitveranstaltungen und in Gesprächen mit Mentorinnen und Mentoren. 


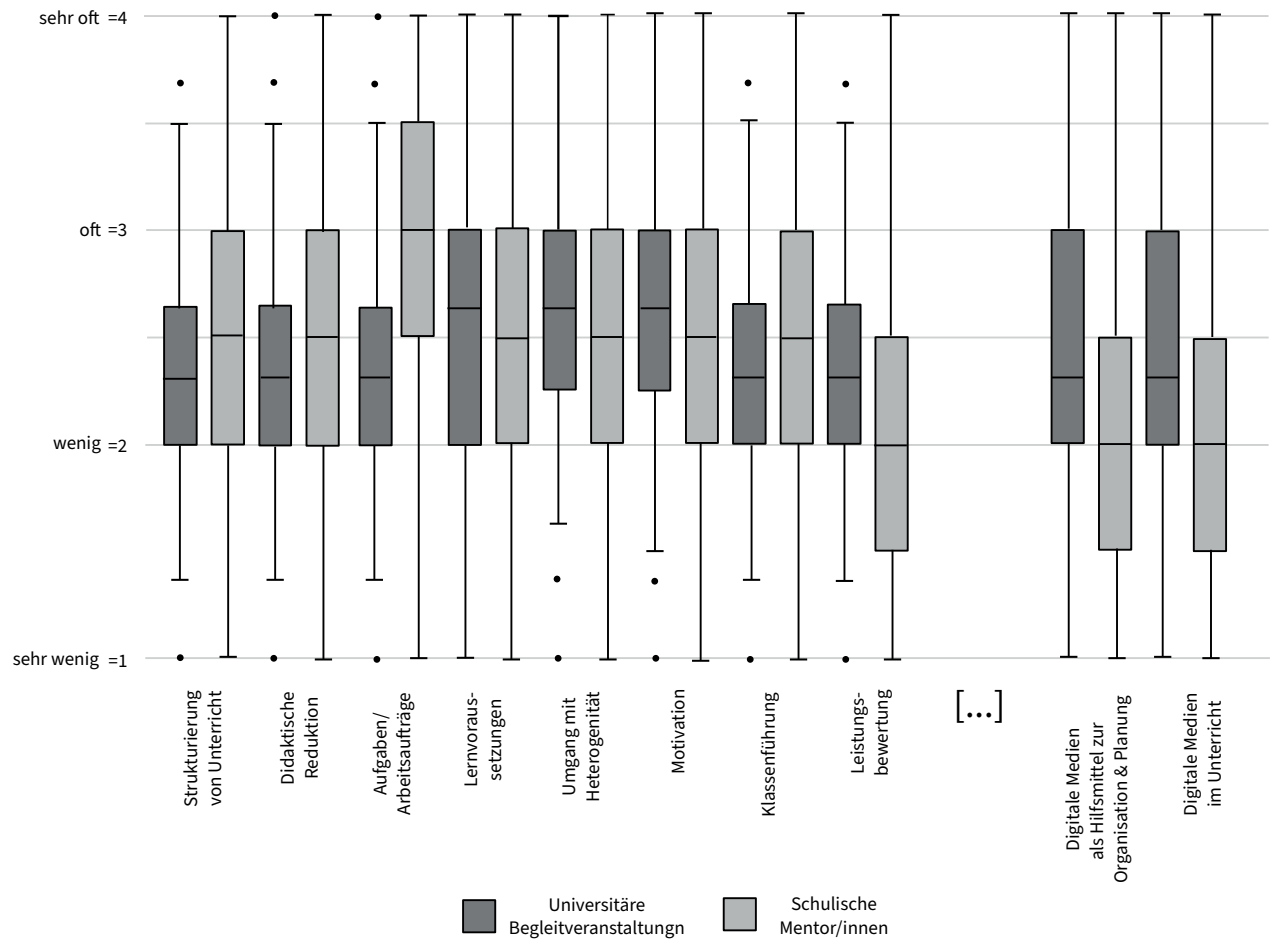

Abb. 10.: Thematisierung von verschiedenen Themenfelder in der universitären und mentoriellen Begleitung ( $\mathrm{N}=325-332$, je nach Item).

Deskriptiv lässt sich feststellen, dass die Behandlung von Themenfeldern mit explizitem Bezug zu digitalen Medien («...als Hilfsmittel zur Organisation und (Unterrichts-)planung» bzw. «...im Unterricht») in den universitären Begleitveranstaltungen vergleichbar zu anderen Themenfeldern wahrgenommen wurde. Eine vergleichsweise geringere Rolle spielten diese Themen in Gesprächen mit Mentorinnen und Mentoren. Zudem wurde die Behandlung digitaler Themen in den Begleitveranstaltungen als etwas intensiver wahrgenommen, als in den Mentorengesprächen.

Um die Thematisierung des digitalen Medieneinsatzes ebenfalls grob zu quantifizieren, wurden auch hier zwei Skalen gebildet, die jeweils nur die beiden Items umfassen: Thematisierung des digitalen Medieneinsatzes in universitären Begleitveranstaltungen (Cronbach's $\alpha=0.91, \mathrm{MW}=2.40, \mathrm{SD}=0.65$ ) und Thematisierung des digitalen Medieneinsatzes in der mentoriellen Begleitung (Cronbach's $\alpha=0.89, \mathrm{MW}=2.05$, $\mathrm{SD}=0.75)$. 


\subsection{Zusammenhänge zwischen Vorerfahrungen und Lerngelegenheiten}

Um Zusammenhänge zwischen den Studierendenvoraussetzungen und Lerngelegenheiten am Lernort Schule sowie am Lernort Universität einzuschätzen, wurden die gebildeten Skalenwerte für alle Studierenden korreliert. Durch paarweisen Ausschluss ist die Anzahl der Studierenden in den jew. Korrelationen unterschiedlich (Tab. 2).

\begin{tabular}{|c|c|c|c|c|c|c|c|c|c|}
\hline & (1) & (2) & (3) & (4) & (5) & (6) & (7) & (8) & (9) \\
\hline $\begin{array}{l}\text { (1) Unterhaltungs- } \\
\text { or. Mediennutzung }\end{array}$ & - & & & & & & & & \\
\hline $\begin{array}{l}\text { (2) Gestaltende } \\
\text { Mediennutzung }\end{array}$ & $\begin{array}{l}.170^{\star *} \\
(\mathrm{~N}=361)\end{array}$ & & & & & & & & \\
\hline $\begin{array}{l}\text { (3) Vorerfahrungen } \\
\text { (Schule) }\end{array}$ & $\begin{array}{c}.111 \\
(\mathrm{~N}=361)\end{array}$ & $\begin{array}{l}.429^{\star \star} \\
(\mathrm{N}=361)\end{array}$ & & & & & & & \\
\hline $\begin{array}{l}\text { (4) Vorerfahrungen } \\
\text { (Studium) }\end{array}$ & $\begin{array}{l}.228^{\star \star} \\
(\mathrm{N}=361)\end{array}$ & $\begin{array}{l}.433^{\star \star} \\
(\mathrm{N}=361)\end{array}$ & $\begin{array}{l}.482^{\star \star} \\
(\mathrm{N}=361)\end{array}$ & & & & & & \\
\hline $\begin{array}{l}\text { (5) Hospitation: } \\
\text { Medien in Präsenz }\end{array}$ & $\begin{array}{c}-.044 \\
(\mathrm{~N}=321)\end{array}$ & $\begin{array}{c}.170 \\
(\mathrm{~N}=321)\end{array}$ & $\begin{array}{c}.006 \\
(N=321)\end{array}$ & $\begin{array}{c}.089 \\
(\mathrm{~N}=321)\end{array}$ & & & & & \\
\hline $\begin{array}{l}\text { (6) Hospitation: } \\
\text { Medien auf Distanz }\end{array}$ & $\begin{array}{c}-.041 \\
(\mathrm{~N}=274)\end{array}$ & $\begin{array}{c}.173 \\
(\mathrm{~N}=274)\end{array}$ & $\begin{array}{c}.135 \\
(\mathrm{~N}=274)\end{array}$ & $\begin{array}{l}.193^{\star \star} \\
(\mathrm{N}=274)\end{array}$ & $\begin{array}{l}.594^{\star \star} \\
(\mathrm{N}=268)\end{array}$ & & & & \\
\hline $\begin{array}{l}\text { (7) Einsatz: Medien } \\
\text { in Präsenz }\end{array}$ & $\begin{array}{c}-.045 \\
(\mathrm{~N}=271)\end{array}$ & $\begin{array}{l}.207^{\star \star} \\
(\mathrm{N}=271)\end{array}$ & $\begin{array}{c}.054 \\
(\mathrm{~N}=271)\end{array}$ & $\begin{array}{c}.155 \\
(\mathrm{~N}=271)\end{array}$ & $\begin{array}{l}.735^{\star \star} \\
(\mathrm{N}=266)\end{array}$ & $\begin{array}{l}.503^{\star *} \\
(\mathrm{~N}=232)\end{array}$ & & & \\
\hline $\begin{array}{l}\text { (8) Einsatz: Medien } \\
\text { auf Distanz }\end{array}$ & $\begin{array}{c}-.137 \\
(\mathrm{~N}=301)\end{array}$ & $\begin{array}{c}.174 \\
(\mathrm{~N}=301)\end{array}$ & $\begin{array}{c}.110 \\
(\mathrm{~N}=301)\end{array}$ & $\begin{array}{c}.144 \\
(\mathrm{~N}=301)\end{array}$ & $\begin{array}{l}.329^{\star \star} \\
(\mathrm{N}=297)\end{array}$ & $\begin{array}{l}.620^{* *} \\
(\mathrm{~N}=258)\end{array}$ & $\begin{array}{l}.519^{\star \star} \\
(\mathrm{N}=262)\end{array}$ & & \\
\hline $\begin{array}{l}\text { (9) Uni-Begleitung: } \\
\text { Medien als Thema }\end{array}$ & $\begin{array}{c}-.060 \\
(\mathrm{~N}=331)\end{array}$ & $\begin{array}{c}.068 \\
(\mathrm{~N}=331)\end{array}$ & $\begin{array}{c}.097 \\
(\mathrm{~N}=331)\end{array}$ & $\begin{array}{c}.012 \\
(\mathrm{~N}=331)\end{array}$ & $\begin{array}{c}.136 \\
(N=314)\end{array}$ & $\begin{array}{c}.188 \\
(\mathrm{~N}=270)\end{array}$ & $\begin{array}{c}.154 \\
(\mathrm{~N}=268)\end{array}$ & $\begin{array}{c}.178 \\
(\mathrm{~N}=297)\end{array}$ & \\
\hline $\begin{array}{l}\text { (10) Mentoren- } \\
\text { Begleitung: Medien } \\
\text { als Thema }\end{array}$ & $\begin{array}{c}-.019 \\
(\mathrm{~N}=324)\end{array}$ & $\begin{array}{c}.156 \\
(\mathrm{~N}=324)\end{array}$ & $\begin{array}{c}.049 \\
(\mathrm{~N}=324)\end{array}$ & $\begin{array}{c}.090 \\
(\mathrm{~N}=324)\end{array}$ & $\begin{array}{l}.504^{\star \star} \\
(\mathrm{N}=310)\end{array}$ & $\begin{array}{l}.312^{\star \star} \\
(\mathrm{N}=267)\end{array}$ & $\begin{array}{l}.459^{\star \star} \\
(\mathrm{N}=266)\end{array}$ & $\begin{array}{l}.247^{\star \star} \\
(\mathrm{N}=294)\end{array}$ & $\begin{array}{l}.306^{\star \star} \\
(\mathrm{N}=322)\end{array}$ \\
\hline
\end{tabular}

Tab. 2.: Zusammenhänge zwischen Vorerfahrungen und Lerngelegenheiten, Korrelationen (Pearson). ${ }^{\star} \mathrm{p}<.005,{ }^{\star *} \mathrm{p}<.001$, alle $\mathrm{p}$-Werte wurden bonferroni-korrigiert.

In der Tabelle sind die Korrelationen hervorgehoben, die auch nach einer Bonferroni-Korrektur mindestens einen signifikanten kleinen Effekt ergaben ( $r>$.2). Ein gestaltendes Mediennutzugsverhalten korreliert positiv mit sehr kleinem Effekt mit dem eigenen Medieneinsatz im Präsenzunterricht. Das Ausmass an beobachtetem Medieneinsatz im Präsenzunterricht korreliert mit mittlerem bis grossem Effekt mit dem Medieneinsatz im eigenen Präsenz- bzw. Distanzunterricht, ebenso diese beiden Skalen untereinander. Bzgl. der Thematisierung digitaler Medien in der Begleitung konnten signifikante Korrelationen zwischen dem Ausmass der Thematisierung in Mentorengesprächen und dem Ausmass des beobachteten und eigenen Medieneinsatzes festgestellt werden.

Um Zusammenhänge zwischen Lerngelegenheiten und der Ausstattung der Schulen zu untersuchen, wurden die vorhandenen digitalen Medien je Person aufsummiert und anschliessend zwei Gruppen mittels Mediansplit gebildet. Dabei fällt die Gruppe mit hoher Ausstattung etwas grösser aus, da sie die Studierenden enthält, 
die genau den Median der Ausstattung angaben. In Tab. 3 sind die Ergebnisse der gebildeten Skalen für beide Gruppen sowie die Ergebnisse einfaktorieller ANOVAs zur Analyse der Gruppenunterschiede dargestellt.

\begin{tabular}{|c|c|c|c|c|c|c|c|c|c|}
\hline & \multicolumn{3}{|c|}{ Niedrige Ausstattung } & \multicolumn{3}{|c|}{ Hohe Ausstattung } & \multirow[b]{2}{*}{$F(1)$} & \multirow[b]{2}{*}{$\mathrm{p}$} & \multirow[b]{2}{*}{$\eta 2$} \\
\hline & $\mathrm{N}$ & MW & SD & $\mathrm{N}$ & MW & SD & & & \\
\hline $\begin{array}{l}\text { Hospitation: Medi- } \\
\text { en in Präsenz }\end{array}$ & 143 & 1.45 & 0.47 & 179 & 1.82 & 0.56 & 38.82 & $<.001$ & .108 \\
\hline $\begin{array}{l}\text { Hospitation: Medi- } \\
\text { en auf Distanz }\end{array}$ & 123 & 1.59 & 0.49 & 152 & 1.87 & 0.61 & 16.86 & $<.001$ & .058 \\
\hline $\begin{array}{l}\text { Einsatz: Medien in } \\
\text { Präsenz }\end{array}$ & 121 & 1.39 & 0.48 & 151 & 1.62 & 0.56 & 12.88 & $<.001$ & .046 \\
\hline $\begin{array}{l}\text { Einsatz: Medien auf } \\
\text { Distanz }\end{array}$ & 132 & 1.28 & 0.49 & 170 & 1.37 & 0.57 & 2.15 & .143 & .007 \\
\hline $\begin{array}{l}\text { Uni-Begleitung: } \\
\text { Medien als Thema }\end{array}$ & 150 & 2.37 & 0.66 & 182 & 2.42 & 0.63 & 0.01 & .944 & .000 \\
\hline $\begin{array}{l}\text { Mentoren-Beglei- } \\
\text { tung: Medien als } \\
\text { Thema }\end{array}$ & 148 & 1.84 & 0.67 & 177 & 2.23 & 0.76 & 23.03 & $<.001$ & .067 \\
\hline
\end{tabular}

Tab. 3.: Zusammenhänge zwischen Lerngelegenheiten und Ausstattung, Mediansplit.

Studierende, die ihr Praxissemester an einer Schule mit vergleichsweise hoher Ausstattung absolvierten, gaben in fast allen Bereichen signifikant höhere Lerngelegenheiten an, mit Ausnahme der Thematisierung digitaler Medien in den universitären Begleitveranstaltungen und dem eigenen Einsatz im Distanzunterricht.

\section{Diskussion}

In diesem Beitrag wurden die Lernvoraussetzungen und -erfahrungen zum Umgang mit digitalen Medien im Unterricht in einer für eine Ausbildungsregion repräsentativen Kohorte Lehramtsstudierender im Praxissemester untersucht, die im Frühjahr 2020 von pandemiebedingten Schulschliessungen betroffen war. Zusammenfassend lässt sich mit Blick auf die Forschungsfragen feststellen, dass die Studierenden über wenige schulische Vorerfahrungen mit digitalen Medien verfügten und im Studium zwar mehr, aber überwiegend Erfahrungen mit Standardanwendungen digitaler Medien gemacht haben. Sie wiesen insgesamt auch ein eher unterhaltungsorientiertes Mediennutzungsverhalten auf. Dies ähnelt Ergebnissen bestehender Untersuchungen (z. B. Schmid et al. 2017; Zawacki-Richter 2015).

Am Lernort Schule gaben die Studierenden wenig Lerngelegenheiten zum Umgang mit digitalen Medien an. Sowohl im hospitierten Präsenz- als auch Distanzunterricht wurden analoge Medien häufiger eingesetzt. Im Distanzunterricht dominierte der Einsatz von E-Mails. Diese Ergebnisse spiegeln das hierzulande generell 
geringe Niveau des Medieneinsatzes im Unterricht (Eickelmann, Bos, und Labusch et al. 2019) sowie den eingeschränkten Einsatz digitaler Medien während der pandemiebedingten Schulschliessungen wieder (Eickelmann und Drossel 2020). Insbesondere im Distanzunterricht haben die Studierenden kaum digitale Medien eingesetzt, wobei beachtet werden muss, dass gut ein Drittel der Studierenden angab, während der Zeit der Schulschliessungen gar nicht am Lernort Schule eingebunden gewesen zu sein. Der digitale Medieneinsatz war zwar in den digital durchgeführten universitären Begleitveranstaltungen durchaus Thema, allerdings weniger in den Gesprächen mit schulischen Mentorinnen und Mentoren.

In Korrelationsanalysen ergab sich, dass die Lerngelegenheiten an beiden Lernorten so gut wie nicht mit dem Nutzungsverhalten und den Vorerfahrungen der Studierenden zusammenhängen. Wohl aber gibt es Korrelationen mit nennenswertem Effekt zwischen den Lerngelegenheiten am Lernort Schule. Mit anderen Worten, Studierende, die den Einsatz digitaler Medien häufiger beobachteten, haben diese häufiger eingesetzt und auch mit Mentorinnen und Mentoren häufiger besprochen. Die Lerngelegenheiten zum Umgang mit digitalen Medien am Lernort Schule scheinen im Wesentlichen von der mentoriellen Begleitung abzuhängen, was generell für das Lernen in Praxisphasen gilt (Clarke, Triggs, und Nielsen 2014).

Bei der Interpretation der Ergebnisse müssen allerdings die Limitationen dieser Untersuchung beachtet werden. Alle Ergebnisse basieren auf Selbstauskünften der Studierenden, die durch fehlerhafte Beobachtung verzerrt sein könnten. Die notwendige Datenbereinigung führte zu teilweise hohen Ausschlüssen von Werten, so dass insbesondere für den Medieneinsatz im eigenen Unterricht die Aussagekraft geringer ist. Ebenso sollte bzgl. der Zusammenhangsanalysen die Unterschiedlichkeit der Skalen berücksichtigt werden, so dass die Ergebnisse ein eher grobes Bild liefern.

Insgesamt zeigt sich, dass der erwartete Möglichkeitsraum für die digitale Professionalisierung nicht ausgeschöpft bzw. genutzt wurde - weder im Sinne einer Professionalisierung der Studierenden für digitale Lehre, noch im Sinne der Unterstützung einer digitalen Innovation durch Studierende an den Praxisschulen. Auch hier erwiesen sich bestimmte Bedingungen, wie die fehlende technische Ausstattung der Schulen als Hinderungsfaktoren. Anzumerken ist, dass bei genauerem Blick schulformspezifische Unterschiede erkennbar sind, die in diesem Beitrag aus Umfangsgründen nicht dargestellt werden konnten. Die Ergebnisse machen insgesamt deutlich, dass ungünstige Professionalisierungsbedingungen am Lernort Schule nur bedingt durch universitäre Anteile der Praxisphase aufgefangen werden konnten. Zukünftig sollte daher die Förderung digitalisierungsbezogener Kompetenzen angehender Lehrpersonen (noch) stärker bereits in der universitären Lehrpersonenbildung berücksichtigt werden. 
Betrachtet man die nach Tondeur et al. (2012) massgeblichen Faktoren für eine gelingende digitale Professionalisierung angehender Lehrpersonen, lassen sich vor dem Hintergrund der Ergebnisse folgende Schlüsse ziehen. Neben der Verzahnung einer theoriebasierten Auseinandersetzung mit Digitalisierung in der Schule, die in der universitären Begleitung zumindest im vergleichbaren Ausmass zu anderen Themenfeldern angesprochen wurde, ist eine hohe Anwendungsorientierung von Vorteil, die sich in den Erfahrungen der Studierenden nicht zeigte; weder als Beobachtende in der Hospitation, noch als eigenständig Gestaltende im Einsatz. Gerade diese Erfahrungen müssen in Zukunft ermöglicht werden. Die Ermöglichung der exemplarischen Gestaltung und Erprobung eigener Lehreinheiten mit digitalen Medien durch angehende Lehrpersonen und ihre Reflexion sollten dabei in allen Ausbildungsbestandteilen von Praxisphasen eine zentrale Rolle spielen. Sie profitieren auch davon, wenn ihre Dozentinnen und Dozenten selbst souverän im Umgang mit digitalen Medien in der universitären Lehre sind, die gewissermassen eine Vorbildfunktion haben. Für die digitale Professionalisierung angehender Lehrpersonen ist daher nicht nur die Beobachtung des digitalen Medieneinsatzes in den schulischen Praxisphasen, sondern auch in den universitären Begleitveranstaltungen ein massgeblicher Faktor. Damit dies gelingen kann, sind letztlich gezielte hochschuldidaktische Fort- und Weiterbildungsmassnahmen für die in der Lehrpersonenbildung tätigen Personen sinnvoll.

Neben den beschriebenen Aspekten auf mikrodidaktischer Ebene sind darüber hinaus aber auch tragfähige Kooperationsbeziehungen zwischen den Akteuren der unterschiedlichen Lernorte, eine gute IT-Infrastruktur und der Zugang zu digitalen Medien (z. B. in Form digitaler Klassenzimmer) sowie die Integration der digitalisierungsbezogenen Förderung in Studienprogrammen, Studienordnungen und Modulbeschreibungen notwendig, um die Förderung digitalisierungsbezogener Kompetenzen angehender Lehrpersonen zu forcieren. Scheiter und Lachner $(2019,547)$ weisen auch darauf hin, dass neben der technischen Infrastruktur, Professionals, die über digitale Kompetenzen verfügen und eine Lehrpersonenbildung, die den Aufbau dieser unterstützt, sowie ein Pool digitaler Materialien und Konzepte zu den zentralen Gelingensbedingungen für ihren lernförderlichen Einsatz im Unterricht zählen.

Obwohl die bildungspolitische Forderung, die digitalisierungsbezogenen Kompetenzen der Lehrenden und Lernenden stärker zu fördern, seit Jahren im Raum steht, kann von einer flächendeckenden Umsetzung bisher nicht die Rede sein. Zwar wurde ab Frühjahr 2020 verstärkt mit öffentlichen Geldern gegengesteuert, allerdings führt auch eine funktionierende technische Ausstattung nicht automatisch zu guten Professionalisierungsbedingungen. Vielmehr spielt auch hier die fundierte medienpädagogische und -didaktische Aus- und Fortbildung von (angehenden) Lehrpersonen eine zentrale Schlüsselrolle (Niesyto 2020, 80-81). Gerade die Schlüsselstrategien auf institutioneller Ebene (Tondeur et al. 2012, 138-42) scheinen noch nicht so umgesetzt zu sein, dass sie sich systematisch positiv auf die mikrodidaktische Ebene auswirken. 
Für die Gestaltung schulpraktischer Studienanteile lässt sich folgern, dass zur Förderung digitaler Professionalisierung zum einen Kooperationsbeziehungen zwischen den Akteuren der unterschiedlichen Lernorte noch aktiver gestaltet und zum anderen begleitende universitäre Veranstaltungen sowohl inhaltlich als auch hinsichtlich der Zielerwartungen bzgl. digitaler Lehrformate konkretisiert werden müssen. Hierbei muss die digitale Professionalisierung auch als gemeinsam geteiltes Ziel der Lehramtsausbildung verstanden werden. Im besten Fall würde ein solches «technology planning and leadership» (Tondeur et al. 2012, 140) auch eine positive Auswirkung auf schulische Lehr-Lern-Prozesse ausserhalb von Praxisphasen der Lehrpersonenbildung haben. Da die Entwicklung der medienbezogenen Anteile der Lehrpersonenausbildung nur langsam vorangeht, ist es kaum verwunderlich, dass die Medienkompetenzen angehender Lehrpersonen eher gering ausgeprägt sind. Zudem fehlt es bisher häufig an einer hinreichenden curricularen Verankerung digitaler Inhalte in der Lehrpersonenausbildung (Bos et al. 2016, 151-52). An deutschen Hochschulen werden z. B. medienpädagogische Studieninhalte überwiegend in Form von integrierten Pflichtveranstaltungen, durch das Angebot medienpädagogischer Studienzertifikate oder Zusatzstudiengänge integriert (Herzig, Aßmann, und Klar 2014). Für ihre nachhaltige Integration ist aber vielmehr eine integrative Hochschulentwicklung notwendig, z. B. die Initiierung von Change Management-Prozessen, die nicht nur die Ebene der Lernumgebung, sondern auch eine systematische Studienprogrammentwicklung und organisationsstrukturelle und prozessuale Faktoren berücksichtigt (Brahm, Jenert, und Euler 2016, 27-33).

Des Weiteren bedarf es nicht nur einer Veränderung der universitären Lehrpersonenausbildung, sondern auch der Erweiterung bestehender Fort- und Weiterbildungsangebote zur gezielten Förderung von digitalen Kompetenzen für Lehrpersonen, damit Studierende am Lernort vermehrt auch Mentorinnen und Mentoren antreffen, die Lerngelegenheiten zum Medieneinsatz ermöglichen. Derzeit sind solche Weiterbildungsangebote eher spärlich vorhanden (Lorenz und Endberg 2019, 62; van Ackeren et al. 2019, 108). In NRW wird dies beispielsweise im Rahmen des BMBF geförderten Verbundprojekts Comeln forciert, das über alle drei Phasen der Lehrpersonenbildung eine stärkere Förderung digitalisierungsbezogener Kompetenzen anstrebt (van Ackeren et al. 2020). 


\section{Literatur}

Ackeren, Isabell van, Heike Buhl, Birgit Eickelmann, Martin Heinrich, und Günther Wolfswinkler. 2020. «Digitalisierung in der Lehrerbildung durch Communities of Practice. Konzeption, Governance und Qualitätsmanagement des Comeln-Verbundvorhabens in NordrheinWestfalen». In Bildung, Schule, Digitalisierung, herausgegeben von Kai Kaspar, Michael Becker-Mrotzek, Sandra Hofhues, Johannes König, und Daniela Schmeinck, 321-26, Münster \& New York: Waxmann. https://doi.org/10.31244/9783830992462.

Ackeren, Isabell van, Stefan Aufenanger, Birgit Eickelmann, Steffen Friedrich, Rudolf Kammerl, Julia Knopf, Kerstin Mayrberger, Heike Scheika, Katharina Scheiter und Mandy SchiefnerRohs. 2019. «Digitalisierung in der Lehrerbildung. Herausforderungen, Entwicklungsfelder und Förderung von Gesamtkonzepten». Die deutsche Schule, 111 (1): 103-19. https://doi. org/10.31244/dds.2019.01.10.

Bertelsmann Stiftung, CHE Centrum für Hochschulentwicklung, Deutsche Telekom Stiftung und Stifterverband für die Deutsche Wissenschaft. 2018. Lehramtsstudium in der digitalen Welt - Professionelle Vorbereitung auf den Unterricht mit digitalen Medien?! Eine Sonderpublikation aus dem Projekt «Monitor Lehrerbildung». https://www.monitor-lehrerbildung. de/export/sites/default/.content/Downloads/Broschuere_Lehrerbildung-in-der-digitalenWelt.pdf.

Bos, Wilfried, Ramona Lorenz, Manuela Endberg, Birgit Eickelmann, Rudolf Kammerl, und Stefan Welling, Hrsg. 2016. Schule digital - der Länderindikator 2016. Kompetenzen von Lehrpersonen der Sekundarstufe I im Umgang mit digitalen Medien im Bundesländervergleich. Münster \& New York: Waxmann. https://www.waxmann.com/index. php?elD=download\&buchnr=3540.

Brahm, Taiga, Tobias Jenert, und Dieter Euler. 2016. «Pädagogische Hochschulentwicklung als Motor für die Qualitätsentwicklung von Studium und Lehre». In Pädagogische Hochschulentwicklung. Von der Programmatik zur Implementierung, herausgegeben von Taiga Brahm, Tobias Jenert, und Dieter Euler,19-36, Wiesbaden: Springer Fachmedien. https:// doi.org/10.1007/978-3-658-12067-2_2.

Clarke, Anthony, Valerie Triggs, und Wendy Nielsen. 2014. "Cooperating Teacher Participation in Teacher Education: A Review of the Literature». Review of Educational Research 84 (2): 163-202. https://doi.org/10.3102/0034654313499618.

Eickelmann, Birgit, Wilfried Bos, und Amelie Labusch. 2019. «Die Studie ICILS 2018 im Überblick - Zentrale Ergebnisse und mögliche Entwicklungsperspektiven». In ICILS 2018 \# Deutschland. Computer- und informationsbezogene Kompetenzen von Schülerinnen und Schülern im zweiten internationalen Vergleich und Kompetenzen im Bereich Computational Thinking, herausgegeben von Birgit Eickelmann, Wilfried Bos, Julia Gerick, Frank Goldhammer, Heike Schaumburg, Knut Schwippert, Martin Senkbeil, Martin, und Jan Vahrenhold, Münster und New York: Waxmann. https://kw.uni-paderborn.de/fileadmin/fakultaet/Institute/erziehungswissenschaft/Schulpaedagogik/ICILS_2018__Deutschland_Berichtsband. pdf. 
Eickelmann, Birgit, und Kerstin Drossel. 2020. Schule auf Distanz. Perspektiven und Empfehlungen für den neuen Schulalltag. Eine repräsentative Befragung von Lehrkräften in Deutschland, herausgegeben von der Vodafone Stiftung. https://www.vodafone-stiftung.de/wpcontent/uploads/2020/05/Vodafone-Stiftung-Deutschland_Studie_Schule_auf_Distanz. pdf.

Fickermann, Detlef, und Benjamin Edelstein. 2020. «Langsam vermisse ich die Schule... Schule während und nach der Corona-Pandemie». DDS - Die Deutsche Schule, Beiheft 16: 9-33. https://doi.org/10.31244/9783830992318.05.

Forsa. 2020. «Corona-Krise: Folgebefragung». Das Deutsche Schulbarometer Spezial. https:// deutsches-schulportal.de/content/uploads/2021/02/Deutsches-Schulbarometer_SpezialFolgebefragung.pdf.

Herzig, Bardo. 2021. «Institutionen der Medienpädagogik: Schule und Medien». In Handbuch Medienpädagogik, herausgegeben von Uwe Sander, Friederike von Gross, und Kai-Uwe Hugger, Wiesbaden: Springer VS. https://doi.org/10.1007/978-3-658-25090-4_95-1.

Herzig, Bardo, Sandra Aßmann, und Tilman-Mathies Klar. 2014. "Grundbildung Medien im Profilstudium im Lehramt». In Grundbildung Medien in pädagogischen Studiengängen, herausgegeben von Peter Imort, und Horst Niesyto, 65-80. München: kopaed.

Herzig, Bardo, und Wiethoff Christoph. 2019. «Konzeptionelle, strukturelle und inhaltliche Gestaltungsaspekte des Praxissemesters an der Universität Paderborn». In Praxissemester (Religion) in NRW: Bilanz und Perspektiven, herausgegeben von Carina Caruso, und Jan Woppowa, https://doi.org/10.17619/UNIPB/1-603.

Huber, Stephan Gerhard, Paula Sophie Günter, Nadine Schneider, Christoph Helm, Marius Schwander, Julia A. Schneider, und Jane Pruitt. 2020. COVID-19 und aktuelle Herausforderungen in Schule und Bildung. Erste Befunde des Schul-Barometers in Deutschland, Österreich und der Schweiz. Münster \& New York: Waxmann.

Huebener, Matthias, C. Katharina Spieß, und Sabine Zinn. 2019. «SchülerInnen in Corona-Zeiten: Teils deutliche Unterschiede im Zugang zu Lernmaterial nach Schultypen und -trägern». DIW Wochenbericht, 47, 866-76. https://doi.org/10.18723/diw_wb:2020-47-1.

Hoffmann, Ilka. 2020. «Die Corona-Pandemie als Katalysator für Schulreformen? Ein persönlicher Blick auf die pädagogische Corona-Praxis». DDS - Die Deutsche Schule, Beiheft 16: 95-101. https://doi.org/10.31244/9783830992318.

KMK. 2016. Strategie der Kultusministerkonferenz «Bildung in der digitalen Welt». https://www. kmk.org/fileadmin/pdf/PresseUndAktuelles/2018/Digitalstrategie_2017_mit_Weiterbildung.pdf.

KMK. 2019a. Ländergemeinsame inhaltliche Anforderungen für die Fachwissenschaften und Fachdidaktiken in der Lehrerbildung. https://www.kmk.org/fileadmin/Dateien/veroeffentlichungen_beschluesse/2008/2008_10_16-Fachprofile-Lehrerbildung.pdf.

KMK. 2019b. Standards für die Lehrerbildung: Bildungswissenschaften. https://www.kmk.org/ fileadmin/veroeffentlichungen_beschluesse/2004/2004_12_16-Standards-LehrerbildungBildungswissenschaften.pdf. 
Krommer, Axel, Pilippe Wampfler, und Wanda Klee. 2020. DISTANZLERNEN. Didaktische Hinweise für Lehrerinnen und Lehrer und Seminarausbilderinnen und Seminarausbilder, herausgegeben vom Ministerium für Schule und Bildung des Landes Nordrhein-Westfalen, https:// www.schulministerium.nrw/system/files/media/document/file/impulspapier_lernen-aufdistanz.pdf.

Lorenz, Ramona, und Manuela Endberg 2019. «Welche professionellen Handlungskompetenzen benötigen Lehrpersonen im Kontext der Digitalisierung in der Schule? Theoretische Diskussion unter Berücksichtigung der Perspektive Lehramtsstudierender». MedienPädagogik (Einzelbeiträge 2019): 61-81. https://doi.org /10.21240/mpaed/00/2019.10.16.X.

Lörz, Markus, Anna Marczuk, Lena Zimmer, Frank Multrus, und Sandra Buchholz. 2020. «Studieren unter Corona-Bedingungen: Studierende bewerten das erste Digitalsemester. Fast 25.000 Studierende aus ganz Deutschland schildern ihre Erfahrungen im digitalen Sommersemester 2020». DZHW-Brief, 05 (2020): 1-8. https://doi.org/10.34878/2020.05.dzhw_brief.

Niesyto, Horst. 2020. «Mobile Digitalmedien in der Primarstufenbildung». In Mobile Medien im Schulkontext, herausgegeben von Dorothee M. Meister, und Ilka Mindt, 79-108. Wiesbaden: Springer VS. https://doi.org/10.1007/978-3-658-29039-9_5.

Persike, Malte, und Julius-David Friedrich. 2016. Lernen mit digitalen Medien aus Studierendenperspektive. Sonderauswertung aus dem CHE Hochschulranking für die deutschen Hochschulen. Berlin: Geschäftsstelle Hochschulforum Digitalisierung.

Prensky, Marc. 2001. «Digital natives, digital immigrants part 1». On the horizon, 9 (5): 1-6. https://doi.org/10.1108/10748120110424816.

Rabe-Matičević, Sandra, und Stefanie Veith. 2020. «Medienbildung in der Hochschule: Handlungsorientierte Medienbildung im Kontext der transformatorischen und kritisch-konstruktiven Bildungstheorie und -didaktik». MedienPädagogik 39 (Orientierungen): 103-20. https://doi.org/10.21240/mpaed/39/2020.12.07.X.

Scheiter, Katharina, und Andreas Lachner. 2019. «DigitalPakt - was nun? Eine Positionierung aus Sicht der Lehr-Lernforschung». Unterrichtswissenschaft, 47: 547-64. https://doi. org/10.1007/s42010-019-00059-2.

Schmid, Ulrich, Lutz Goertz, Sabine Radomski, Sabrina Thom, und Julia Behrens. 2017. Monitor Digitale Bildung. Die Hochschulen im digitalen Zeitalter. https://www.bertelsmann-stiftung.de/fileadmin/files/BSt/Publikationen/GrauePublikationen/DigiMonitor_Hochschulen_final.pdf.

Sommer, Michael. 2020. «Eine respektable Notlösung. Ergebnisse einer Umfrage zum «CoronaSemester»». Forschung \& Lehre, Nr. 8. https://www.forschung-und-lehre.de/lehre/hochschullehrer-beklagen-verschlechterung-der-lehre-2939.

Tondeur, Jo, Johan van Braak, Guoyuan Sang, Joke Voogt, Petra Fisser, und Anne OttenbreitLeftwich. 2012. "Preparing pre-service teachers to integrate technology in education: A synthesis of qualitative evidence». Computers and Education, 59: 134-44. https://doi. org/10.1016/j.compedu.2011.10.009. 
Traus, Anna, Katharina Höffken, Severine Thomas, Katharina Mangold, und Wolfgang Schröer, Wolfgang. 2020. Stu.diCo. - Studieren digital in Zeiten von Corona. Erste Ergebnisse der bundesweiten Studie Stu.diCo. https://doi.org/10.18442/150.

Vogelsang, Christoph, Alexander Finger, Daniel Laumann, und Christoph Thyssen. 2019. «Vorerfahrungen, Einstellungen und motivationale Orientierungen als mögliche Einflussfaktoren auf den Einsatz digitaler Werkzeuge im naturwissenschaftlichen Unterricht». Zeitschrift für Didaktik der Naturwissenschaften, 25: 115-29, https://doi.org/10.1007/s40573-01900095-6.

Wilde, Mathias, Said D. Werner, Barbara Gumbmann, und Solveigh Hieronimus. 2020. «Hochschulen, Corona und jetzt?» In Future Skills Diskussionspapier 4, herausgegeben vom Stifterveband. https://www.stifterverband.org/medien/hochschulen-corona-und-jetzt.

Wößmann, Ludger, Vera Freundl, Elisabeth Grewenig, Philipp Lergetporer, Katharina Werner, und Larissa Zierow. 2020. «Bildung in der Coronakrise: Wie haben die Schulkinder die Zeit der Schulschließungen verbracht, und welche Bildungsmaßnahmen befürworten die Deutschen?»Ifo Schnelldienst, 73: 25-39 https://www.ifo.de/DocDL/sd-2020-09-woessmannetal-bildungsbarometer-corona.pdf.

Zawacki-Richter, Olaf. 2015. «Zur Mediennutzung im Studium - unter besonderer Berücksichtigung heterogener Studierender». Zeitschrift für Erziehungswissenschaft, 18(3): 527-549. https://doi.org/10.1007/s11618-015-0618-6. 